\title{
JPSS-1 VIIRS Radiometric Characterization and Calibration Based on Pre-Launch Testing
}

\author{
Hassan Oudrari ${ }^{1, *}$, Jeff McIntire ${ }^{1}$, Xiaoxiong Xiong ${ }^{2}$, James Butler ${ }^{2}$, Qiang Ji ${ }^{1}$, \\ Thomas Schwarting ${ }^{1}$, Shihyan Lee ${ }^{1,+}$ and Boryana Efremova ${ }^{1, \ddagger}$ \\ Received: 29 October 2015; Accepted: 28 December 2015; Published: 6 January 2016 \\ Academic Editors: Changyong Cao, Richard Müller and Prasad S. Thenkabail \\ 1 Science Systems and Applications, Inc., Lanham, MD 20706, USA; jeffrey.mcintire@ssaihq.com (J.M.); \\ qiang.ji@ssaihq.com (Q.J.); thomas.schwarting@ssaihq.com (T.S.); shihyan.lee@nasa.gov (S.L.); \\ boryana.efremova@noaa.gov (B.E.) \\ 2 NASA Goddard Space Flight Center, Greenbelt, MD 20771, USA; xiaoxiong.xiong-1@nasa.gov (X.X.); \\ james.j.butler@nasa.gov (J.B.) \\ * Correspondence: Hassan.oudrari-1@nasa.gov; Tel.: +1-301-867-2094; Fax: +1-301-867-2151 \\ $\dagger$ Current affiliation: SAIC, Beltsville, MD 20705, USA. \\ $\ddagger$ Current affiliation: Earth Resources Technology, Inc., Silver Spring, MD 20707, USA.
}

\begin{abstract}
The Visible Infrared Imaging Radiometer Suite (VIIRS) on-board the first Joint Polar Satellite System (JPSS) completed its sensor level testing on December 2014. The JPSS-1 (J1) mission is scheduled to launch in December 2016, and will be very similar to the Suomi-National Polar-orbiting Partnership (SNPP) mission. VIIRS instrument has 22 spectral bands covering the spectrum between 0.4 and $12.6 \mu \mathrm{m}$. It is a cross-track scanning radiometer capable of providing global measurements twice daily, through observations at two spatial resolutions, $375 \mathrm{~m}$ and $750 \mathrm{~m}$ at nadir for the imaging and moderate bands, respectively. This paper will briefly describe J1 VIIRS characterization and calibration performance and methodologies executed during the pre-launch testing phases by the government independent team to generate the at-launch baseline radiometric performance and the metrics needed to populate the sensor data record (SDR) Look-Up-Tables (LUTs). This paper will also provide an assessment of the sensor pre-launch radiometric performance, such as the sensor signal to noise ratios (SNRs), radiance dynamic range, reflective and emissive bands calibration performance, polarization sensitivity, spectral performance, response-vs-scan (RVS), and scattered light response. A set of performance metrics generated during the pre-launch testing program will be compared to both the VIIRS sensor specification and the SNPP VIIRS pre-launch performance.
\end{abstract}

Keywords: JPSS; SNPP; VIIRS; pre-launch; radiometric; performance; calibration; spectral

\section{Introduction}

The purpose of this paper is to provide an overview of the initial assessment of the Visible Infrared Imaging Radiometer Suite (VIIRS) sensor onboard the first Joint Polar Satellite System (JPSS-1 or J1). This VIIRS instrument is the second flight unit of its series, and it has a similar design to the first sensor that was launched on 28 October 2011 on-board the Suomi-National Polar-Orbiting Partnership (SNPP) satellite. SNPP VIIRS has been sending high quality remote sensing data for about four years [1,2], and has not only contributed to the continuity of climate data record initiated by other spaceborne Earth observing instruments, but has also supported many crucial environmental, economic and scientific applications [3-5]. The JPSS program will ensure the launch of multiple VIIRS instruments over the next two decades, starting with J1 in December 2016.

VIIRS is a scanning radiometer that collects visible and infrared imagery and radiometric measurements of the Earth's surface to generate environmental data records (EDRs) in support of 
data continuity initiated by heritage sensors such as the Advanced Very High Resolution Radiometer (AVHRR), the Sea-viewing Wide Field-of-view Sensor (SeaWiFS) and the Moderate Resolution Imaging Spectroradiometer (MODIS) [6]. Products derived from VIIRS will support key applications and research studies such as weather forecasting and global measurements of atmospheric, oceanic, and land surface variables [7-9].

J1 VIIRS sensor pre-launch results summarized in this paper are based on the data analyses performed by the VIIRS Characterization Support Team (VCST), with close interaction with other government and contractor teams, including the National Aeronautics and Space Administration (NASA) flight project and science teams, the Aerospace Corporation team, the National Oceanic and Atmospheric Administration (NOAA)-Center for Satellite Applications and Research (STAR) team, and the University of Wisconsin team.

In the following sections, we will provide an overview of the key J1 VIIRS pre-launch radiometric and spectral performance assessments, describing briefly the methodologies used, and comparing the results to SNPP VIIRS pre-launch performance as well as to the sensor requirements. The VIIRS spatial performance assessments are not included in this paper. J1 VIIRS design and testing program will be presented in Section 2. The summary of VIIRS pre-launch testing and sensor performance will be presented in Section 3 for all reflective solar bands (RSB) and thermal emissive bands (TEB), focusing on key performance metrics such as the signal-to-noise (SNR), dynamic range, polarization sensitivity, relative spectral response, and scattered light contamination. A summary and conclusion of J1 VIIRS pre-launch radiometric and spectral performance as well as future performance enhancements will be presented in the last section, Section 4.

\section{Sensor Design and Testing Program}

\subsection{J1 Sensor Design}

The VIIRS sensor is designed to operate in a polar, sun-synchronous orbit with a nominal altitude of $828 \mathrm{~km}$ at an inclination angle of approximately 98 degrees relative to the equator (1:30 PM local equatorial crossing time, ascending node) [10]. It collects radiometric and imaging data in 22 spectral bands covering the visible and infrared spectral region between 0.4 to $12.6 \mu \mathrm{m}$ (Table 1). The moderate resolution bands (M-bands) and imaging resolution bands (I-bands) have a spatial resolution of $\sim 750 \mathrm{~m}$ and $\sim 375$ m respectively, while the ground swath is $\sim 3040 \mathrm{~km}$, permitting daily global coverage.

J1 VIIRS has the same design as SNPP VIIRS with similar on-board calibrators [11]: the Spectralon ${ }^{\circledR}$ solar diffuser (SD) to calibrate the RSB, the solar diffuser stability monitor (SDSM) to track the SD spectral degradation, the V-groove blackbody (BB) to calibrate the TEB, and the space view (SV) to be used for background subtraction (Figure 1).

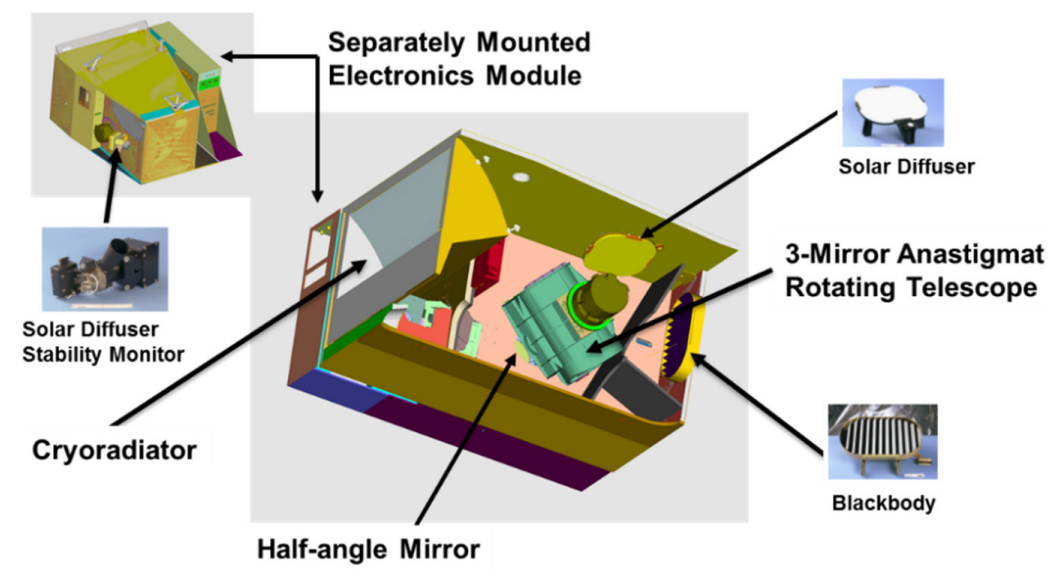

Figure 1. VIIRS instrument design and on-board calibrators: Solar Diffuser (SD), Solar Diffuser Stability Monitor (SDSM), and blackbody (BB). 
Table 1. VIIRS 22 bands characteristics and key requirements.

\begin{tabular}{|c|c|c|c|c|c|c|c|c|c|}
\hline \multicolumn{10}{|c|}{ Reflective Solar Bands (RSB) } \\
\hline $\begin{array}{l}\text { Band } \\
\text { Name }\end{array}$ & Gain & $\begin{array}{l}\text { Center } \\
\text { Wavelength } \\
\text { (nm) }\end{array}$ & $\begin{array}{l}\text { Bandwidth } \\
(\mathrm{nm})\end{array}$ & $\begin{array}{c}\text { Focal } \\
\text { Plane } \\
\text { Assembly }\end{array}$ & $\begin{array}{l}\text { FOV (km) } \\
\text { Nadir/EOS }\end{array}$ & $\begin{array}{l}\text { Polarization } \\
\text { Sensitivity } \\
(\%)\end{array}$ & $\mathbf{L}_{\text {typ }}$ & $\mathbf{L}_{\min } / \mathbf{L}_{\max }$ & SNR \\
\hline DNB & VG & 700 & 400 & DNB & $0.8 / 1.6$ & NS & 3 e-5 $\left(^{*}\right)$ & $3 e-5 / 200\left(^{*}\right)$ & 6 \\
\hline M1 & $\begin{array}{l}\text { HG } \\
\text { LG }\end{array}$ & 412 & 20 & VISNIR & $0.8 / 1.6$ & 3 & $\begin{array}{l}44.9 \\
155\end{array}$ & $\begin{array}{c}30 / 135 \\
135 / 615\end{array}$ & $\begin{array}{l}352 \\
316\end{array}$ \\
\hline M2 & $\begin{array}{l}\text { HG } \\
\text { LG }\end{array}$ & 445 & 18 & VISNIR & $0.8 / 1.6$ & 2.5 & $\begin{array}{c}40 \\
146\end{array}$ & $\begin{array}{c}26 / 127 \\
127 / 687\end{array}$ & $\begin{array}{l}380 \\
409\end{array}$ \\
\hline M3 & $\begin{array}{l}\text { HG } \\
\text { LG }\end{array}$ & 488 & 20 & VISNIR & $0.8 / 1.6$ & 2.5 & $\begin{array}{c}32 \\
123\end{array}$ & $\begin{array}{c}22 / 107 \\
107 / 702\end{array}$ & $\begin{array}{l}416 \\
414\end{array}$ \\
\hline M4 & $\begin{array}{l}\text { HG } \\
\text { LG }\end{array}$ & 555 & 20 & VISNIR & $0.8 / 1.6$ & 2.5 & $\begin{array}{l}21 \\
90\end{array}$ & $\begin{array}{c}12 / 78 \\
78 / 667\end{array}$ & $\begin{array}{l}362 \\
315\end{array}$ \\
\hline M5 & $\begin{array}{l}\text { HG } \\
\text { LG }\end{array}$ & 672 & 20 & VISNIR & $0.8 / 1.6$ & 2.5 & $\begin{array}{l}10 \\
68\end{array}$ & $\begin{array}{l}8.6 / 59 \\
59 / 651\end{array}$ & $\begin{array}{l}242 \\
360\end{array}$ \\
\hline $\begin{array}{c}\text { I1 } \\
\text { M6 }\end{array}$ & $\begin{array}{l}\text { SG } \\
\text { SG }\end{array}$ & $\begin{array}{l}640 \\
746\end{array}$ & $\begin{array}{l}80 \\
15\end{array}$ & $\begin{array}{l}\text { VISNIR } \\
\text { VISNIR }\end{array}$ & $\begin{array}{l}0.4 / 0.8 \\
0.8 / 1.6\end{array}$ & $\begin{array}{l}2.5 \\
2.5\end{array}$ & $\begin{array}{l}22 \\
9.6\end{array}$ & $\begin{array}{l}5 / 718 \\
5.3 / 41\end{array}$ & $\begin{array}{l}119 \\
199\end{array}$ \\
\hline M7 & $\begin{array}{l}\text { HG } \\
\text { LG }\end{array}$ & 865 & 39 & VISNIR & $0.8 / 1.6$ & $\begin{array}{l}3 \\
3\end{array}$ & $\begin{array}{c}6.4 \\
33.4\end{array}$ & $\begin{array}{l}3.4 / 29 \\
29 / 349\end{array}$ & $\begin{array}{l}215 \\
340\end{array}$ \\
\hline I2 & SG & 865 & 39 & VISNIR & $0.4 / 0.8$ & 3 & 25 & $10.3 / 349$ & 150 \\
\hline M8 & SG & 1240 & 20 & SMWIR & $0.8 / 1.6$ & NS & 5.4 & $3.5 / 164.9$ & 74 \\
\hline M9 & SG & 1378 & 15 & SMWIR & $0.8 / 1.6$ & NS & 6 & $0.6 / 77.1$ & 83 \\
\hline M10 & SG & 1610 & 60 & SMWIR & $0.8 / 1.6$ & NS & 7.3 & $1.2 / 71.2$ & 342 \\
\hline $\mathrm{I} 3$ & SG & 1610 & 60 & SMWIR & $0.4 / 0.8$ & NS & 7.3 & $1.2 / 72.5$ & 6 \\
\hline M11 & SG & 2250 & 50 & SMWIR & $0.8 / 1.6$ & NS & 0.12 & $0.12 / 31.8$ & 10 \\
\hline \multicolumn{10}{|c|}{ Thermal Emissive Bands (TEB) } \\
\hline $\begin{array}{l}\text { Band } \\
\text { Name }\end{array}$ & Gain & $\begin{array}{l}\text { Center } \\
\text { Wavelength } \\
(\mathrm{nm})\end{array}$ & $\begin{array}{l}\text { Bandwidth } \\
\text { (nm) }\end{array}$ & $\begin{array}{c}\text { Focal } \\
\text { Plane } \\
\text { Assembly }\end{array}$ & $\begin{array}{l}\text { FOV (km) } \\
\text { Nadir/EOS }\end{array}$ & $\begin{array}{l}\text { Polarization } \\
\text { Sensitivity } \\
\quad(\%)\end{array}$ & $\mathrm{T}_{\mathrm{typ}(\mathrm{K})}$ & $\mathrm{T}_{\min } / \mathrm{T}_{\max (\mathrm{K})}$ & $\operatorname{NEdT}_{(K)}$ \\
\hline I4 & SG & 3740 & 380 & SMWIR & $0.4 / 0.8$ & NS & 270 & $210 / 353$ & 2.5 \\
\hline M12 & SG & 3700 & 180 & SMWIR & $0.8 / 1.6$ & NS & 270 & $230 / 353$ & 0.396 \\
\hline M13 & HG & 4050 & 155 & SMWIR & $0.8 / 1.6$ & $\begin{array}{l}\text { NS } \\
\text { NS }\end{array}$ & 300 & $230 / 343$ & 0.107 \\
\hline M14 & SG & 8550 & 300 & LWIR & $0.8 / 1.6$ & NS & 270 & $\begin{array}{l}343 / 634 \\
190 / 336\end{array}$ & $\begin{array}{l}0.423 \\
0.091\end{array}$ \\
\hline M15 & SG & 10763 & 1000 & LWIR & $0.8 / 1.6$ & NS & 300 & $190 / 343$ & 0.07 \\
\hline I5 & SG & 11450 & 1900 & LWIR & $0.4 / 0.8$ & NS & 210 & $190 / 340$ & 1.5 \\
\hline M16 & SG & 12013 & 950 & LWIR & $0.8 / 1.6$ & NS & 300 & $190 / 340$ & 0.072 \\
\hline
\end{tabular}

VIIRS design has incorporated multiple enhancements based on lessons learned from heritage sensors such as SNPP VIIRS and MODIS. The light collected by the rotating telescope assembly (RTA) is distributed to three focal planes, the Visible Near-Infrared (VisNIR), the Short- and Mid-Wave Infrared (SMWIR), and the Long-Wave Infrared (LWIR). All bands in the LWIR focal planes are thermally controlled at about $80.5 \mathrm{~K}$, and all bands in the SMWIR are floating very close to the LWIR FPA temperature. Light reaching the focal planes' detectors is converted into analog electric signal then digitized through analog-to-digital conversion. The VIIRS telescope scans the Earth within \pm 56.1 degree of nadir, then the blackbody at about 100 degrees, the SD at about 159 degrees, and the SV at about -65 degrees, in sequence.

Based on lessons learned from SNPP program, a few key changes were made to the J1 instrument to enhance its performance, including: (1) replaced the VIIRS 1394 communication bus with SpaceWire to resolve the anomalies observed by SNPP on-orbit [1,2]; (2) replaced the single board computer with a new design to resolve the computer lock-up issue observed by SNPP [1,2]; (3) the coating on the RTA mirrors was changed from Nickel (Ni) to a proprietary process (VQ) to enhance spatial stability with temperature; (4) the dichroic 2 coating was redesigned to correct the focus between SMWIR and LWIR bands; (5) the proper RTA mirror coating process was used to avoid Tungsten contamination [1,2] and (6) the VisNIR integrated filter assembly was redesigned to significantly reduce filter optical scatter and out-of-band (OOB) features [11]. 


\subsection{J1 Sensor Testing Program}

With continuous support from the government independent team, J1 VIIRS instrument was put through a very intensive ground test program, to ensure proper characterization and calibration, to understand the instrument performance, to use reliable and approved data analysis methods, and to investigate, close and document all anomalies identified during testing. The test setup graphs are not shown in this paper since these are considered company property. The VIIRS test program, led by the sensor contractor Raytheon, provided a comprehensive sensor characterization and performance assessment over the full range of instrument operating conditions that will be encountered on orbit. It has also provided the calibration and characterization values needed for each band, detector, gain state, half-angle mirror (HAM) side, electronics side, and instrument temperature. VIIRS general test plan covers three (3) key pre-launch phases: ambient (August 2013-January 2014), sensor level thermal vacuum (TV) (July-October 2014) and spacecraft level TV (expected in the spring of 2016).

By the end of sensor level testing, a large set of performance test procedures were executed to characterize the instrument under various environments (ambient and/or TV), to support the verification of sensor performance requirements [12], and to simulate long-term on-orbit performance. Key sensor performance testing was completed at three (3) instrument plateaus, cold, nominal and hot, during TV testing to cover the range of expected on-orbit conditions. J1 VIIRS key performance testing is listed in Table 2.

Table 2. Key sensor performance testing during ambient and thermal vacuum testing phases.

\begin{tabular}{cc}
\hline \multicolumn{2}{c}{ Ambient Phase Testing August 2013-January 2014 } \\
\hline Polarization sensitivity & Near Field Response (NFR) \\
Response vs Scan (RVS) & Stray Light Response (SLR) \\
Radiometric characterization & Electrical and Dynamic Crosstalk \\
\hline Thermal Vacuum Testing July 2014-November 2014 \\
\hline RSB and TEB calibration & Relative Spectral Response in-band \& OOB ${ }^{1}$ \\
Radiometric stability & Band-to-Band Registration (BBR) \\
Gain transition determination & Line Spread Function (LSF) \\
\hline
\end{tabular}

${ }^{1}$ The VisNIR RSR was derived in ambient when the sensor was in the TV chamber (with the chamber door open).

Early in the J1 test program, two (2) important decisions were made that defined how the instrument will be operated: (1) set the cold focal planes temperature at $80.5 \mathrm{~K}$ based on the thermal balance testing performed at TV hot plateau; (2) and designated the VIIRS electronics side A as the primary electronics, even though both sides (A and B) showed comparable performance.

\section{J1 VIIRS Pre-Launch Performance}

An overview of J1 performance assessment will be described in this section based on the NASA VCST team test data analysis. Comparison to sensor specification and SNPP VIIRS will be presented as well. Methodologies will be briefly described for each performance metric, but in general, data analysis methodologies are quite similar to those used for SNPP VIIRS [11].

A series of key VIIRS performance tests were performed at ambient, TV, or both [12]. Because of limited space, this paper will focus on key radiometric performance metrics including the J1 radiometric calibration for the RSB and TEB, dynamic range, signal to noise ratio (SNR), noise equivalent differential temperature (NEdT), polarization sensitivity, relative spectral response (RSR), response versus scan-angle (RVS), near field response (NFR) and stray light response (SLR). All of the calibration parameters and SNR were derived in the TV environment at three instrument temperature plateaus: cold, nominal and hot plateau. In this paper, only the performance for electronics side A the nominal plateau (i.e., closest to on-orbit configuration) will be reported. 


\subsection{RSB Radiometric Calibration}

J1 VIIRS RSB were carefully calibrated in a TV environment using a National Institute of Standards and Technology (NIST) traceable light source, the $100 \mathrm{~cm}$ diameter spherical integrating source (SIS100). Another source, the three mirror collimator (TMC) SIS, capable of reaching high radiance values was also used to calibrate M1-M3 low gain. While the SIS100 has a radiance monitor to track the source fluctuations and drifts, the TMC does not, and therefore it is considered less accurate than the SIS100. In addition, the space view source (SVS) was used to collect the dark offset needed to generate the background corrected detector response or digital number $(d n)$.

The detector response is related to the light source radiance $\mathrm{L}\left(\mathrm{Wm}^{-2} \cdot \mathrm{sr}^{-1} \cdot \mu \mathrm{m}^{-1}\right)$ through a second degree polynomial:

$$
L=c_{0}+c_{1} \cdot d n+c_{2} \cdot d n^{2}+\mathrm{U}\left(d n^{3}\right)
$$

where $c_{0}, c_{1}$, and $c_{2}$ are the calibration coefficients and $\mathrm{U}\left(d n^{3}\right)$ denotes the truncation error.

The calibration coefficients can be determined from Equation (1) through curve fitting to unsaturated measurements at different radiance levels over the band's dynamic range $\left[\mathrm{L}_{\min }, \mathrm{L}_{\max }\right]$; however, the instability of the source over time can lead to high uncertainties for the radiometric calibration algorithm. To mitigate the impact of the source instability, the radiance measurements were performed with and without an attenuator screen inserted into the optical path between the SIS100 and VIIRS RTA entrance aperture. The attenuator screen is an opaque plate with small holes to allow a determined fraction of light through (transmittance of $\sim 56 \%$ ). The time between attenuator in and out is very short to minimize the effect of source instability on the measurements ( $2 \mathrm{~min})$.

Consequently, the ratio of the detected spectral radiances with and without the attenuator is equal to the attenuator transmittance $(\tau)$, as:

$$
\tau=\frac{\frac{c_{0}}{c_{1}}+d n_{\text {in }}+\left(\frac{c_{2}}{c_{1}}\right) d n_{\text {in }}^{2}}{\frac{c_{0}}{c_{1}}+d n_{\text {out }}+\left(\frac{c_{2}}{c_{1}}\right) d n_{\text {out }}^{2}}
$$

where $\mathrm{dn}_{\text {in }}$ and $\mathrm{dn}_{\text {out }}$ denote the $d n$ with and without the attenuator, respectively. A 3-sigma outlier rejection criteria is used during the calculation.

To facilitate the data regression, Equation (2) is rewritten as:

$$
h_{0}(\tau-1)+\left(\tau d n_{\text {out }}-d n_{\text {in }}\right)+h_{2}\left(\tau d n_{\text {out }}^{2}-d n_{\text {in }}^{2}\right)=0
$$

where $h_{0}=c_{0} / c_{1}$, and $h_{2}=c_{2} / c_{1}$.

The model parameters $\tau, h_{0}$, and $h_{2}$ are determined through a non-linear least-square process. It is worth noting that the RSB on-orbit calibration is performed through the linear coefficient $\left(c_{1}\right)$ updates, and this coefficient or gain is determined pre-launch by inverting Equation (1) and averaging over the selected source levels:

$$
c_{1}=\left\langle\frac{L}{h_{0}+d n_{\text {out }}+h_{2} d n_{\text {out }}^{2}}\right\rangle
$$

The RSB radiometric calibration coefficients are derived in TV at the cold, nominal, and hot temperature plateaus for each detector, HAM side, gain stage, and electronic side, and are then implemented into the SDR LUTs to support on-orbit calibration [13].

In general, the band averaged $c_{0} / c_{1}$ coefficient is very small and is on the order of $10^{-1}\left(c_{0}\right.$ varies within \pm 0.4 ). Exceptions include M1-M3 low gain and SWIR bands M8-M11, where $c_{0} / c_{1}$ has values up to 3. The high values could be explained by the TMC inaccuracies noted previously, and the SWIR non-linearity issue described in a sub-section below. The $c_{0} / c_{1}$ 's variation over the three TV temperature plateaus and across detectors as well as its uncertainty is small, and is in general on the order of $10^{-1}$, except M1-M3 low gain which has uncertainty values up to 7, probably due to the TMC 
fluctuations. The quadratic term $c_{2} / c_{1}$ has mean values on the order of $10^{-6}$ for all RSB bands, and its uncertainty is also on the order of $10^{-6}$. The average gain, $c_{1}$, shows some sensitivity to instrument temperature, varying by up to $5 \%$ between TV plateaus, while its uncertainty is very small, on the order of $10^{-4}$.

\subsubsection{SNR Calculation and Performance}

SNR was computed for each detector using the SIS100 radiance at various levels to cover the dynamic range of each RSB. This SNR is derived for each sample position (i.e., angular position in scan) by dividing the cross-scan average by their standard deviation as:

$$
S N R=\frac{1}{M} \sum_{j=1}^{j=M}\left[\frac{\frac{1}{N}\left[\sum_{i=1}^{i=N} d n_{i . j}\right]}{\sigma_{j}}\right]
$$

where $\mathrm{M}, \mathrm{N}$ are the total number of samples and scans respectively, and $i, j$ are the scan and sample number respectively. The SNR processing approach is based on the cross-scan standard deviation $\left(\sigma_{j}\right)$, which avoids the spatial non-uniformity when viewing the SIS aperture across samples.

The SNR estimates derived at each SIS level were fit using a mathematical formula described by Equation (6) in order to compute the SNR at any radiance level, and to smooth out the variability in the SNR over radiance levels:

$$
S N R=\frac{L}{\sqrt{k_{0}+k_{1} L+k_{2} L^{2}}}
$$

The band averaged SNR values are shown in Table 3. All J1 RSB meet the SNR specification at $\mathrm{L}_{\text {typ }}$ with good margins, and overall, these margins are better than those measured for SNPP. It is also worth noting that having a contamination-free RTA mirror coating, J1 SNR performance is expected to be even better than SNPP at the end of mission life. The smallest and largest SNR margins for $\mathrm{J} 1$ are $51 \%$ and $3172 \%$ for M2 high gain and I3, respectively. It is also worth mentioning that the I3 detector 4 is a very noisy detector $(S N R=6.7$ ), and its responsivity is about $50 \%$ lower than the other detectors in this band. This detector is being studied to mitigate striping features in I3 images and associated products.

Table 3. J1 VIIRS SNR (at $\mathrm{L}_{\text {typ }}$ ) and $\mathrm{L}_{\text {sat }}$ values for RSB, and comparison to sensor specification and $\mathrm{SNPP}$. The ratio between measured performance and specification (Spec) is also shown. For dual gain

\begin{tabular}{|c|c|c|c|c|c|c|c|c|c|c|c|}
\hline Band & $\begin{array}{l}\text { Gain } \\
\text { Stage }\end{array}$ & $\begin{array}{l}\text { SNR } \\
\text { (Spec) }\end{array}$ & $\begin{array}{l}\text { Lmax } \\
\text { (Spec) }\end{array}$ & $\begin{array}{l}\text { SNPP } \\
\text { SNR }\end{array}$ & J1 SNR & $\begin{array}{c}\text { SNPP } \\
\text { SNR/Spec }\end{array}$ & $\begin{array}{c}\text { J1 } \\
\text { SNR/Spec }\end{array}$ & $\begin{array}{c}\text { SNPP } \\
\text { L }_{\text {sat }}\end{array}$ & $J 1 L_{\text {sat }}$ & $\begin{array}{c}\text { SNPP } \\
\mathrm{L}_{\text {sat }} / \text { Spec }\end{array}$ & $\begin{array}{c}\mathrm{J} 1 \\
\mathrm{~L}_{\mathrm{sat}} / \text { Spec }\end{array}$ \\
\hline M1 & High & 352 & 135 & 613 & 636 & 1.74 & 1.81 & 172 & 154 & 1.27 & 1.14 \\
\hline M1 & Low & 316 & 615 & 1042 & 1066 & 3.30 & 3.37 & 696 & 705 & 1.13 & 1.15 \\
\hline M2 & Low & 409 & 687 & 963 & 986 & 2.35 & 2.41 & 827 & 880 & 1.20 & 1.28 \\
\hline M3 & High & 416 & 107 & 683 & 706 & 1.64 & 1.70 & 125 & 113 & 1.17 & 1.06 \\
\hline M3 & Low & 414 & 702 & 1008 & 1063 & 2.44 & 2.57 & 843 & 838 & 1.20 & 1.19 \\
\hline M5 & High & 242 & 59 & 373 & 380 & 1.54 & 1.57 & 66 & 61 & 1.12 & 1.04 \\
\hline M5 & Low & 360 & 651 & 776 & 751 & 2.16 & 2.09 & 726 & 725 & 1.12 & 1.11 \\
\hline M6 & High & 199 & 41 & 409 & 428 & 2.06 & 2.15 & 48 & 48 & 1.16 & 1.16 \\
\hline M7 & High & 215 & 29 & 524 & 549 & 2.44 & 2.55 & 31 & 31 & 1.07 & 1.06 \\
\hline M7 & Low & 340 & 349 & 721 & 760 & 2.12 & 2.23 & 414 & 409 & 1.19 & 1.17 \\
\hline M8 & High & 74 & 164.9 & 358 & 335 & 4.84 & 4.53 & 126 & 118 & 0.77 & 0.72 \\
\hline M9 & High & 83 & 77.1 & 290 & 325 & 3.49 & 3.91 & 84 & 80 & 1.09 & 1.04 \\
\hline I3 & High & 6 & 72.5 & 176 & 190 & 29.36 & 31.72 & 70 & 66 & 0.97 & 0.91 \\
\hline
\end{tabular}
bands, the $\mathrm{L}_{\text {sat }}$ of high gain stage represents the radiance transition $\left(\mathrm{L}_{\text {trans }}\right)$ to low gain. 


\subsubsection{Dynamic Range}

J1 RSB dynamic range was also verified, and RSB saturation was found to be comparable to SNPP pre-launch performance, meeting the specification with acceptable margins, except for bands M8 (72\%) and I3 (91\%). These two $\mathrm{L}_{\max }$ non-compliances were expected (similar to SNPP) and have a limited effect on environmental and science products based on SNPP experience. As a note, SNPP M8 and I3 saturation became compliant post-launch because of the RTA mirror degradation on-orbit (decreasing radiometric sensitivity), but J1 will not have that benefit since the mirror coating issue causing this radiometric degradation was corrected in the J1 flight hardware. For dual gain bands, the high gain $\mathrm{L}_{\text {sat }}$ shown in Table 3 represents the radiance transition $\left(\mathrm{L}_{\text {trans }}\right)$ from high gain to low gain. The requirement for $\mathrm{L}_{\text {trans }}$ is to be within $+50 \%$ above $\mathrm{L}_{\max }$. As shown in Table 3, all dual gain bands are compliant with this requirement, with $\mathrm{M} 1$ having the highest transition, $14 \%$ higher than specified high gain $\mathrm{L}_{\max }$.

\subsubsection{RSB Calibration Uncertainties}

VIIRS RSB requirements on the absolute radiometric calibration are against the spectral reflectance accuracy of $2 \%$ when viewing a uniform scene of typical radiance. This uncertainty verification was performed using contributors which have been constrained by sensor-level requirements while others are based on the sensor contractor allocations [14]. A key contributor to the radiometric uncertainty includes the detector response characterization uncertainty, which states that the response of a detector to a range of radiance levels from $\mathrm{L}_{\min }$ to $\mathrm{L}_{\max }$ must be fit with a second degree polynomial to within $0.3 \%$. This characterization uncertainty requirement was very challenging for many VIIRS RSB to meet, but the spectral reflectance accuracy (2\%) was met for all RSB except M11 (2.25\%) [14], primarily due to the uncertainty of the SD bi-directional reflectance factor (BRF) at SWIR wavelengths.

\subsubsection{SWIR Linearity Issue}

J1 SWIR M-bands exhibited more non-linear behavior at low radiance than what was observed on SNPP. Figure 2 shows the ratios of attenuator out signal to attenuator in signal for SWIR bands M8-M11. The non-constant dn ratio reflects the large non-linear response at low radiance levels, while SNPP dn ratios were much flatter. An investigation of this issue discovered that an analog signal processor bias voltage for the SMWIR focal plane was set to a different voltage value $(-0.2 \mathrm{~V})$ compared to its setting on SNPP $(-0.4 \mathrm{~V})$. The non-linearity present in the SWIR M-bands produces large errors in the attenuator ratios, and therefore large errors in the radiance characterization uncertainty and uniformity. Quantized data are also observed at low radiance values and are harder to address in the calibration algorithm. A mitigation plan is being prepared to enhance the calibration performance of the SWIR bands, based on either a third degree polynomial equation or the adoption of a two-piece calibration (i.e., calibration over two radiance ranges).
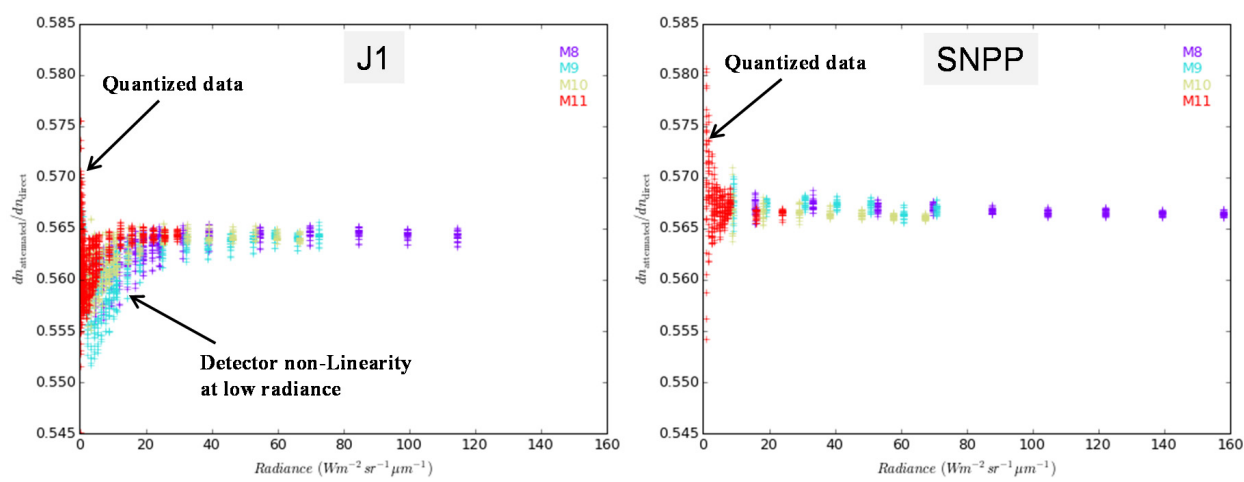

Figure 2. Comparison of J1 and SNPP SWIR (M8-M11) non-linearity as shown by the ratio of sensor response value $(\mathrm{dn})$ for attenuator in and out measurements. 


\subsubsection{DNB Performance and Linearity Issue}

While J1 DNB performance was measured using similar testing as the M-bands and I-bands, additional testing was performed to enhance the DNB characterization (at low light). Data analysis during first baseline testing at cold plateau showed a larger than expected non-linear response for DNB high gain A and B (HGA, HGB). The mid-gain and low gain stages also showed non-linearity features, but to a much lower extent. Upon further analysis, linear fit residuals plotted on a detector basis showed increasing residuals for high gain stages from aggregation modes 21 up to 32 . There are 32 aggregation modes that are used by the DNB charge-coupled device (CCD) when scanning from beginning to end of scan. Since the CCD is a matrix of detectors in the scan and track directions, the aggregation consist of averaging a certain number of pixels (averaging is performed in the electronics) in the scan and track directions to keep the spatial resolution almost constant throughout the scan. The root cause of this DNB non-linearity issue was associated to two incorrect biases set in the focal plane interface electronics coupling into the feedthrough effect, and resulting in charge bleeding from one detector to the next (crosstalk) on the signal output line between successive acquisition frames.

Figure 3 shows the DNB response (dn) for HGB (HGA shows similar features) at four aggregation modes (1, 21, 26 and 32), where the non-linearity is clearly increasing from aggregation mode 21 up to aggregation 32 . This non-linearity also increases as the radiance decreases. This issue was intensively investigated, and a solution referenced as Option 21 was identified to significantly reduce the non-linearity effect, which consists of fixing the aggregation mode from zone 21 outward, meaning that all aggregation zones from 21 to 32 will be assigned aggregation mode 21. It is also important to note that reducing the non-linearity effect led to better radiometric uncertainty, uniformity (striping) between detectors and between aggregation zones, and SNR performance. The only caveat for this solution is the loss of ability to maintain constant spatial resolution at large scan angles, but this is considered as low risk compared to the non-linearity effect.
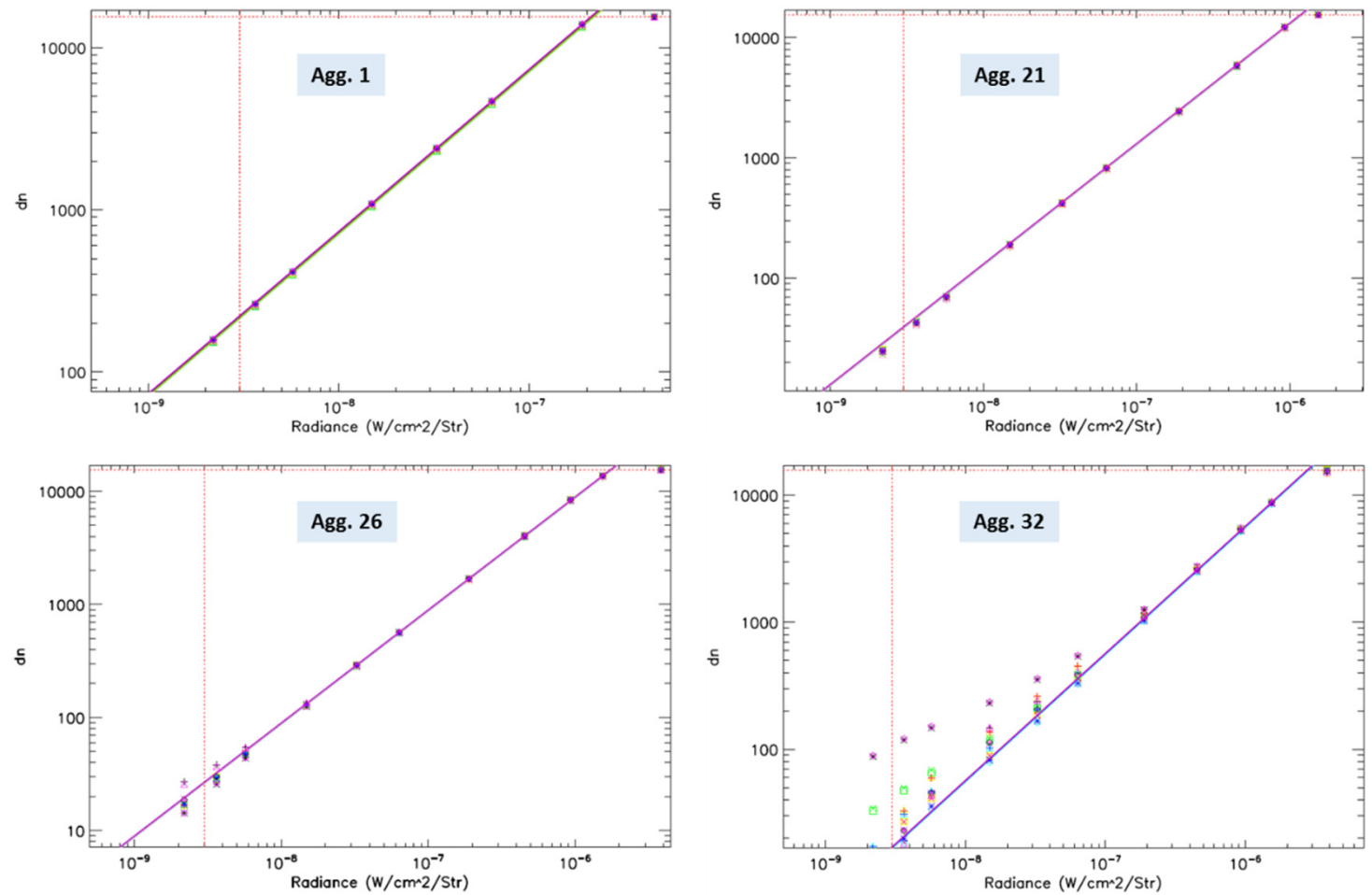

Figure 3. J1 DNB HGB response (dn) as a function of radiance in aggregation zones 1, 21, 26 and 32. Non-linearity feature starts to increase noticeably between aggregation modes 21 and 32 at low radiance. The 16 DNB detectors are represented by different symbols. 
Based on Option 21 configuration used to correct for non-linearity, the DNB SNR showed compliance with specification at $\mathrm{L}_{\min }$ for all aggregation zones, and the DNB dynamic range is compliant with $\mathrm{L}_{\max }$ specification, $200 \mathrm{Wm}^{-2} \cdot \mathrm{sr}^{-1}$, except for aggregation zone $1\left(\sim 97 \%\right.$ of $\left.\mathrm{L}_{\max }\right)$. This is considered as low risk for the DNB since more than $99.9 \%$ of the Earth targets seen by SNPP DNB (based on data from 14 orbits) have radiance values less than $150 \mathrm{Wm}^{-2} \cdot \mathrm{sr}^{-1}$.

Further concerns associated with on-orbit calibration, and how the non-linearity could impact the radiance quality are being investigated. Additional DNB testing is planned at spacecraft TV level (April 2016) to derive at-launch offset tables based on the new optimized sample-to-zone mapping, and to enhance DNB on-orbit calibration performance.

\subsection{Thermal Emissive Bands (TEB) Calibration}

J1 VIIRS thermal band calibration is referenced to a NIST traceable external blackbody calibration source (BCS). The radiance reaching the detector is the sum of the source radiance as well as contributors along the optical path (i.e., the RTA, HAM, and aft optics). The reflectance factors represent the total reflectance of the RTA mirrors, HAM and aft-optics. The RVS is the scan angle dependent relative reflectance of the HAM. The temperature of each source is determined from one or more thermistors, and the radiances of the sources are determined via Planck's law convolved over the RSR of each spectral band over the extended band-pass [15].

The path difference radiance between the two sources (BCS and SV) is then:

$$
L_{B C S}=R V S_{B C S} \varepsilon_{B C S} L_{B C S}-\frac{\left(R V S_{B C S}-R V S_{S V}\right)}{\rho_{R T A}}\left[L_{H A M}-\left(1-\rho_{R T A}\right) L_{R T A}\right]
$$

The path difference radiance is modeled as a quadratic polynomial in the offset corrected digital response, or

$$
L=c_{0}+c_{1} d n+c_{2} d n^{2}
$$

The retrieved EV radiance for the BCS is determined by inverting Equation (7), or

$$
L_{B C S-r e t}=\frac{\left(c_{0}+c_{1} d n_{B C S}+c_{2} d n_{B C S}^{2}\right)}{R V S_{B C S}}+\frac{\left(R V S_{B C S}-R V S_{S V}\right)}{R V S_{B C S} \rho_{R T A}}\left[L_{H A M}-\left(1-\rho_{R T A}\right) L_{R T A}\right]
$$

The low gain state of band M13 is calibrated using a second high temperature external blackbody, the TMC blackbody. The calibration of the TMC blackbody was tied to the BCS by cross calibration at scene temperatures where both sources overlap.

\section{NEdT Calculation}

The NEdT is the fluctuation in the scene temperature equivalent to the system noise and is computed via the equation

$$
N E d T=\frac{N E d L}{\frac{\partial L}{\partial T}}=\frac{L_{B C S}}{S N R \frac{\partial L}{\partial T}}
$$

The derivative is of Planck's law with respect to the source temperature. The NEdT was determined at all source levels and compared to the specified value at $\mathrm{T}_{\text {typ }}$; this was determined by fitting the SNR as a function of path difference radiance. As shown in Table 4, all TEB band average and per detectors NEdT meet the specification with good margins, and the performance is comparable to SNPP. A mild dependence of NEdT to sensor temperature (TV plateaus) was observed. The smallest and greatest NEdT margins were observed for M14 (39\%) and I4 (595\%) respectively. Detector noise variability is small in general, with the exception of detector 4 in M15 and detector 5 in M16B which are out-of-family detectors, and could lead to striping in some data products.

Table 4 also shows the J1 maximum temperature and comparison to the specification and SNPP. All bands have saturation values above the specified $\mathrm{T}_{\max }$, and comparable to SNPP. For J1, digital saturation occurred first for all bands, while for SNPP two bands, M12 and M14, exhibit analog 
saturation before digital saturation. The TEB saturation values are consistent among electronics sides and temperature plateaus to within $3 \mathrm{~K}$. M13 low gain saturation was not observed in TV testing, but saturation is expected to be around $670 \mathrm{~K}$ based on ambient testing, while SNPP VIIRS has shown saturation around $654 \mathrm{~K}$.

Table 4. J1 VIIRS $\mathrm{T}_{\max }$ and NEdT performance for TEB derived in TV at nominal plateau, and comparison to Spec and SNPP.

\begin{tabular}{ccccccc}
\hline \multirow{2}{*}{ Band } & \multicolumn{3}{c}{$\mathbf{T}_{\text {max }}$} & \multicolumn{3}{c}{ NEdT at $\mathbf{T}_{\text {typ }}$} \\
\cline { 2 - 7 } & Spec & SNPP & J1 & Spec & SNPP & J1 \\
\hline I4 & 353 & 357 & 357 & 2.5 & 0.41 & 0.42 \\
I5 & 340 & 373 & 370 & 1.5 & 0.42 & 0.41 \\
M12 & 353 & 357 & 358 & 0.396 & 0.13 & 0.12 \\
M13 HG & 343 & 364 & 363 & 0.107 & 0.044 & 0.043 \\
M13 LG & 634 & - & - & 0.423 & 0.340 & 0.304 \\
M14 & 336 & 347 & 348 & 0.091 & 0.061 & 0.050 \\
M15 & 343 & 365 & 359 & 0.070 & 0.030 & 0.026 \\
M16 & 340 & 368 & 369 & 0.072 & 0.038 & 0.043 \\
\hline
\end{tabular}

The radiometric response uniformity (RRU) represents the detector-to-detector uniformity (or striping) and is quantified by the following equation:

$$
R R U=\frac{\left|L_{B C S-r e t}-\left\langle L_{B C S-r e t}\right\rangle_{D}\right|}{N E d L}
$$

where the average EV retrieved radiance is over all detectors in a band and NEdL is derived from Equation (10). The sensor specification is met if the RRU is less than unity within the radiance range from $\mathrm{L}_{\min }$ to $0.9 \mathrm{~L}_{\max }$.

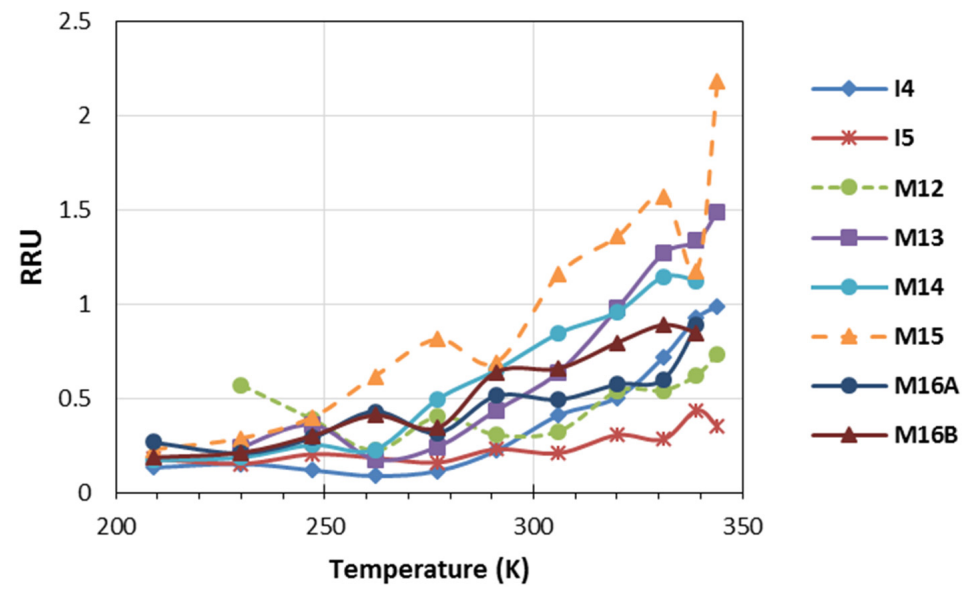

Figure 4. J1 maximum RRU as a function of source (BCS) temperature is shown for all TEB, HAM 0, and nominal plateau (RRU is compliant if less than unity).

Figure 4 shows the detector-to-detector striping performance represented by the RRU metric as a function of scene temperature for all emissive bands. We can easily see that the risk of striping increases with temperature for all TEB bands, because the deviation of the detector retrieved radiance from the band average increases with temperature while the NEdL levels off. Results derived per HAM side, electronics side and temperature plateau have shown some performance variations, with RRU for some bands reaching up to 2 at highest temperatures (larger than $310 \mathrm{~K}$ ). We should also emphasize the difficulty to meet this requirement for both SNPP and J1 since VIIRS TEB has a very good noise performance (very low NEdL). 
The absolute radiance difference (ARD) is the percent difference between the retrieved and calculated BCS radiances, and it is essentially a measure of the fit uncertainty's effect on the accuracy of the retrieved radiance, or

$$
A R D=100 \frac{L_{B C S}-r e t-L_{B C S}}{L_{B C S}}
$$

The band averaged ARD for J1 TEB derived at nominal plateau are shown in Table 5 alongside the specified values in parenthesis. As expected, the ARD results show excellent thermal calibration for all bands at the specified temperatures, and the only exception is M12 at very low temperature $(230 \mathrm{~K})$ where the ARD value of 7.6 is slightly higher than the specified value of 7.0. The temperature errors converted from the ARD values are also shown in Table 5 at each specified temperature. The corresponding temperature uncertainty was computed by taking the difference in temperature derived from the radiance with and without the radiance uncertainty. Overall, the ARD performance (and temperature errors) is comparable between J1 and SNPP.

Table 5. J1 Band averaged ARD (\%) at specified temperature shown for all TEB, HAM 0, and nominal plateau. ARD specification values are in parenthesis. In addition, shown in the lower part of this table are the corresponding temperature errors $(\mathrm{K})$.

\begin{tabular}{|c|c|c|c|c|c|c|c|c|}
\hline \multicolumn{9}{|c|}{ ARD Performance (\%) } \\
\hline Temp (K) & I4 & I5 & M12 & M13 & M14 & M15 & M16A & M16B \\
\hline 190 & $\sim$ & $\sim$ & $\sim$ & $\sim$ & $\begin{array}{c}0.68 \\
(12.30)\end{array}$ & $\begin{array}{c}0.29 \\
(2.10)\end{array}$ & $\begin{array}{c}0.17 \\
(1.60)\end{array}$ & $0.25(1.60)$ \\
\hline 230 & $\sim$ & $\sim$ & $\begin{array}{c}7.60 \\
(7.00)\end{array}$ & $\begin{array}{l}2.95 \\
(5.7)\end{array}$ & $\begin{array}{c}0.11 \\
(2.40)\end{array}$ & $\begin{array}{c}0.07 \\
(0.60)\end{array}$ & $\begin{array}{c}0.08 \\
(0.60)\end{array}$ & $0.04(0.60)$ \\
\hline 267 & $\begin{array}{c}0.48 \\
(5.00)\end{array}$ & $\begin{array}{c}0.10 \\
(2.50)\end{array}$ & $\sim$ & $\sim$ & $\sim$ & $\sim$ & $\sim$ & $\sim$ \\
\hline 270 & $\sim$ & $\sim$ & $\begin{array}{c}0.24 \\
(0.70)\end{array}$ & $\begin{array}{c}0.15 \\
(0.70)\end{array}$ & $\begin{array}{c}0.08 \\
(0.60)\end{array}$ & $\begin{array}{c}0.05 \\
(0.40)\end{array}$ & $\begin{array}{c}0.04 \\
(0.40)\end{array}$ & $0.04(0.40)$ \\
\hline 310 & $\sim$ & $\sim$ & $\begin{array}{c}0.25 \\
(0.70)\end{array}$ & $\begin{array}{c}0.17 \\
(0.70)\end{array}$ & $\begin{array}{c}0.11 \\
(0.40)\end{array}$ & $\begin{array}{c}0.06 \\
(0.40)\end{array}$ & $\begin{array}{c}0.03 \\
(0.40)\end{array}$ & $0.04(0.40)$ \\
\hline 340 & $\sim$ & $\sim$ & $\begin{array}{c}0.27 \\
(0.70)\end{array}$ & $\begin{array}{c}0.18 \\
(0.70)\end{array}$ & $\begin{array}{c}0.09 \\
(0.50)\end{array}$ & $\begin{array}{c}0.05 \\
(0.40)\end{array}$ & $\begin{array}{c}0.03 \\
(0.40)\end{array}$ & $0.03(0.40)$ \\
\hline \multicolumn{9}{|c|}{ Temperature Error (K) } \\
\hline Temp (K) & I4 & I5 & M12 & M13 & M14 & M15 & M16A & M16B \\
\hline 190 & $\sim$ & $\sim$ & & $\sim$ & 0.2 & 0.08 & 0.07 & 0.1 \\
\hline 230 & $\sim$ & $\sim$ & 1.01 & 0.71 & 0.04 & 0.02 & 0.03 & 0.02 \\
\hline 267 & 0.13 & 0.05 & $\sim$ & $\sim$ & $\sim$ & $\sim$ & $\sim$ & $\sim$ \\
\hline 270 & $\sim$ & $\sim$ & 0.05 & 0.04 & 0.04 & 0.03 & 0.04 & 0.03 \\
\hline 310 & $\sim$ & $\sim$ & 0.06 & 0.05 & 0.07 & 0.04 & 0.03 & 0.04 \\
\hline 340 & $\sim$ & $\sim$ & 0.08 & 0.08 & 0.08 & 0.04 & 0.04 & 0.04 \\
\hline
\end{tabular}

\subsection{Polarization Sensitivity}

During ambient phase testing, J1 polarization sensitivity was characterized using an integrating sphere combined with a sheet polarizer at seven different scan angles in the 400 to $900 \mathrm{~nm}$ range covering all VisNIR bands [16,17]. The sheet polarizer was mounted on a rotary stage and was rotated in 15 degree increments from 0 to 360 degrees. Additionally, a long wavelength spectral blocking filter was placed in the optical path when measuring the short wavelength bands, M1 to M3, to eliminate near infrared OOB contributions. The polarization sensitivity was derived for all VisNIR bands, detectors, HAM sides, and seven scan angles $(-55,-45,-20,-8,22,45,55)$, using Fourier series. The quality of J1 measurements based on the Fourier transform is good, since only the zeroth and second order terms have shown non-negligible results. The polarization sensitivity factors derived based on ambient testing have revealed unexpectedly large non-compliance for four bands, M1-M4. This polarization issue was linked to the redesigned VisNIR spectral filters, and confirmed by the sensor polarization 
model $[16,17]$. Based on these findings further performance testing was conducted in the post-TV phase adding four more scan-angles $(-37,-30,-15,4)$ and limited testing with monochromatic laser source for two bands, M1 and M4, to verify the quality of the sensor polarization modeling [16,17]. The monochromatic polarization testing confirmed the large diattenuation on both edges of the filters bandpass causing the large polarization sensitivity seen in the broadband testing.

The final J1 mean degree of linear polarization (DoLP) values are shown for all VisNIR bands in Table 6 for HAM side 1. The polarization maximum and specification values per band are also shown. The linear polarization sensitivity for bands M1 to M4 was higher than the specified limit, with maximums (both HAM sides) as high as $\sim 6.42 \%$ for $\mathrm{M} 1, \sim 4.36 \%$ for $\mathrm{M} 2, \sim 3.08 \%$ for $\mathrm{M} 3$, and $\sim 4.35 \%$ for M4. Differences in linear polarization sensitivity with HAM side are as high as $\sim 1 \%$, where HAM 1 is generally larger.

Table 6. DoLP (HAM 1) for both SNPP and J1 sensors. The maximum polarization is for both HAM sides. Numbers in bold represent performance non-compliance.

\begin{tabular}{|c|c|c|c|c|c|c|c|c|c|c|c|c|c|c|}
\hline \multirow{2}{*}{ Band } & \multirow{2}{*}{ Sensor } & \multicolumn{11}{|c|}{ Scan Angle } & \multirow{2}{*}{$\begin{array}{c}\text { Max } \\
\text { Pol. }\end{array}$} & \multirow{2}{*}{ Spec } \\
\hline & & -55 & -45 & -37 & -30 & -20 & -15 & -8 & 4 & 22 & 45 & 55 & & \\
\hline \multirow[b]{2}{*}{ I1 } & SNPP & 0.86 & 0.76 & $\sim$ & $\sim$ & 0.62 & $\sim$ & 0.59 & $\sim$ & 0.54 & 0.58 & 0.61 & 1.24 & 2.5 \\
\hline & $\mathrm{J} 1$ & 0.86 & 0.9 & 0.95 & 0.95 & 0.94 & 0.98 & 0.95 & 0.98 & 1 & 1.03 & 1.04 & 1.03 & 2.5 \\
\hline \multirow{2}{*}{ I2 } & SNPP & 0.49 & 0.45 & $\sim$ & $\sim$ & 0.47 & $\sim$ & 0.51 & $\sim$ & 0.56 & 0.56 & 0.55 & 0.56 & 3 \\
\hline & $\mathrm{J} 1$ & 1.19 & 0.92 & 0.75 & 0.62 & 0.5 & 0.51 & 0.48 & 0.5 & 0.53 & 0.58 & 0.61 & 0.92 & 3 \\
\hline \multirow{2}{*}{ M1 } & SNPP & 3.14 & 2.73 & $\sim$ & $\sim$ & 2.01 & $\sim$ & 1.83 & $\sim$ & 1.45 & 1.23 & 1.39 & 2.73 & 3 \\
\hline & $\mathrm{J} 1$ & 5.57 & 5.73 & 5.86 & 6.06 & 6.17 & 6.19 & 6.34 & 6.41 & 6.42 & 6.17 & 5.96 & 6.42 & 3 \\
\hline \multirow[b]{2}{*}{ M2 } & SNPP & 2.25 & 2.05 & $\sim$ & $\sim$ & 1.65 & $\sim$ & 1.54 & $\sim$ & 1.28 & 1.17 & 1.3 & 2.05 & 2.5 \\
\hline & $\mathrm{J} 1$ & 4.08 & 4.08 & 4.13 & 4.23 & 4.18 & 4.18 & 4.23 & 4.25 & 4.19 & 4.36 & 4.46 & 4.36 & 2.5 \\
\hline \multirow[b]{2}{*}{ M3 } & SNPP & 1.45 & 1.31 & $\sim$ & $\sim$ & 0.96 & $\sim$ & 0.85 & $\sim$ & 0.62 & 0.71 & 0.81 & 1.31 & 2.5 \\
\hline & $\mathrm{J} 1$ & 2.92 & 2.86 & 2.83 & 2.85 & 2.76 & 2.74 & 2.75 & 2.74 & 2.85 & 3.08 & 3.11 & 3.08 & 2.5 \\
\hline \multirow[b]{2}{*}{ M4 } & SNPP & 1.59 & 1.52 & $\sim$ & $\sim$ & 1.37 & $\sim$ & 1.3 & $\sim$ & 1.02 & 0.86 & 0.82 & 1.52 & 2.5 \\
\hline & $\mathrm{J} 1$ & 4.03 & 4.2 & 4.33 & 4.35 & 4.32 & 4.35 & 4.3 & 4.29 & 4.15 & 3.99 & 3.91 & 4.35 & 2.5 \\
\hline \multirow[b]{2}{*}{ M5 } & SNPP & 0.81 & 0.74 & $\sim$ & $\sim$ & 0.7 & $\sim$ & 0.69 & $\sim$ & 0.61 & 0.59 & 0.57 & 1.02 & 2.5 \\
\hline & $\mathrm{J} 1$ & 2.1 & 2.17 & 2.23 & 2.19 & 2.13 & 2.14 & 2.07 & 2.03 & 2.02 & 1.99 & 1.97 & 2.23 & 2.5 \\
\hline \multirow{2}{*}{ M6 } & SNPP & 1.29 & 1.14 & $\sim$ & $\sim$ & 0.96 & $\sim$ & 0.92 & $\sim$ & 0.81 & 0.75 & 0.7 & 1.14 & 2.5 \\
\hline & $\mathrm{J} 1$ & 1.03 & 0.92 & 0.89 & 0.87 & 0.86 & 0.91 & 0.91 & 0.95 & 0.96 & 0.95 & 0.94 & 1.32 & 2.5 \\
\hline \multirow[b]{2}{*}{ M7 } & SNPP & 0.52 & 0.47 & $\sim$ & $\sim$ & 0.43 & $\sim$ & 0.44 & $\sim$ & 0.48 & 0.47 & 0.45 & 0.48 & 3 \\
\hline & $\mathrm{J} 1$ & 1.18 & 0.92 & 0.74 & 0.61 & 0.48 & 0.47 & 0.43 & 0.46 & 0.47 & 0.52 & 0.56 & 0.92 & 3 \\
\hline
\end{tabular}

Large detector-to-detector and scan angle variations were observed within various bands (Figure 5), and these variations could reach up to $\sim 4 \%$ (M1). This is likely the result of angle of incidence changes on the VisNIR spectral filter assembly. The final uncertainty analysis has demonstrated compliance with uncertainty specification $(0.5 \%)$, with an overall polarization factor uncertainty less than $0.22 \%$ for all bands except M1 which showed an uncertainty of about $0.37 \%$. While the DNB has no requirement for polarization sensitivity, data analysis has shown this polarization performance to vary between $1.36 \%$ and $1.6 \%$ over the scan-angle range, detectors and HAM sides.

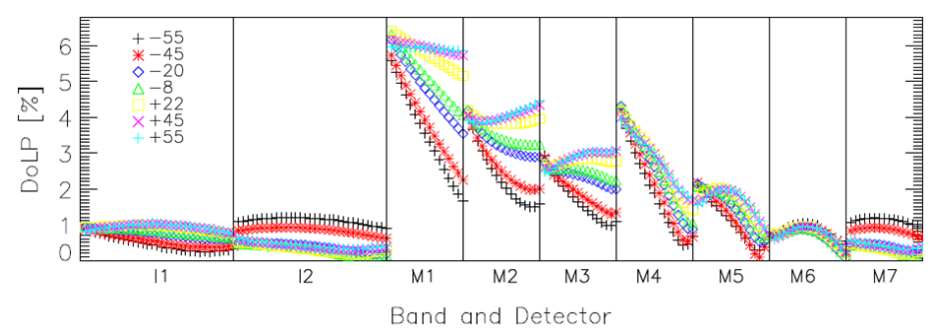

Figure 5. The DoLP (HAM 1) per detector and across scan angles. 
Figure 6 shows a comparison of the DoLP results derived for M1 and M4 bands, based on the broadband source and the monochromatic source measurements [16,17]. In general, the results agree well, and the difference is less than $0.5 \%$ for the DoLP and less than 14 degrees for the phase. These measurement results were also compared to the outputs of the J1 polarization model. Our preliminary assessment is that the model was not able to represent the detector dependence with the accuracy needed, especially for M4 (maximum difference $\sim 1.5 \%$ ), and therefore, our recommendation was to revise the model and its component inputs for $\mathrm{J} 1$ and future instruments (J2+).

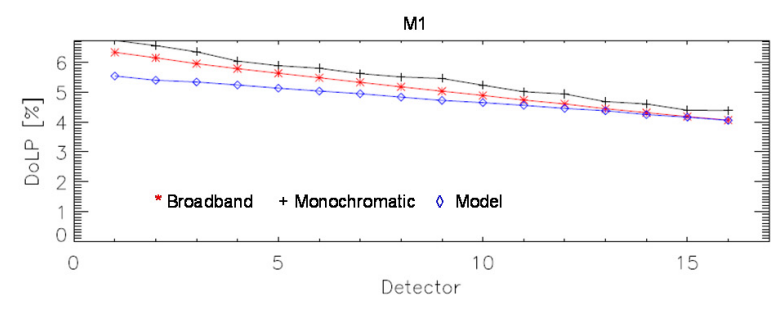

M1

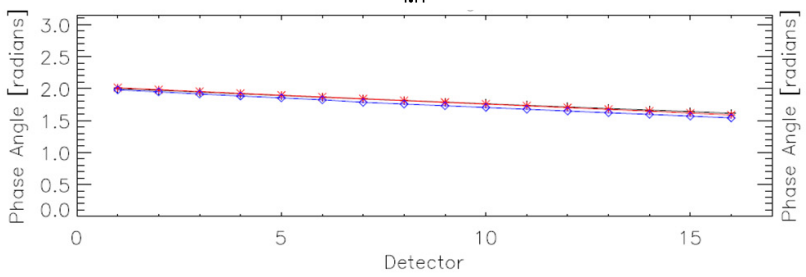

M4

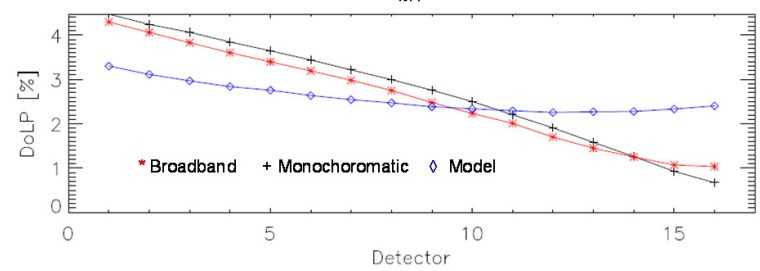

M4

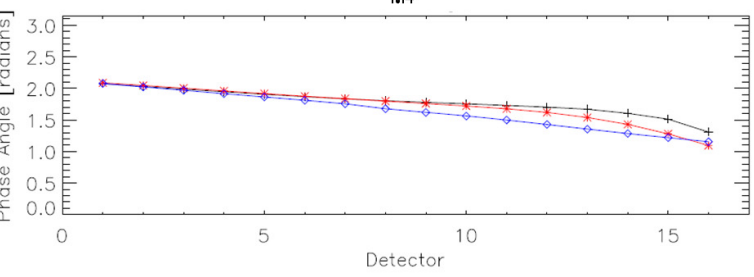

Figure 6. J1 DoLP and phase angle for M1 and M4, using broadband $\left(^{*}\right)$, monochromatic (+), and sensor model $(\diamond)$.

\subsection{Relative Spectral Response (RSR)}

VIIRS sensor level spectral testing was performed using a double monochromator source, the Spectral Measurement Assembly (SpMA) in ambient for the VisNIR bands, and in the TV environment at nominal plateau for the SMWIR and LWIR bands [18]. The monochromator exit slit image was aligned to illuminate one spectral band at a time, and the spectral response was measured over a spectral region covering roughly the acceptance of the dichroic spectral bandpass. The VIIRS spectral data were corrected for the spectral shape of the source using a reference detector data set and normalized to the peak response to determine the VIIRS RSR. The data were analyzed to determine each band's center wavelength, Full Width Half Maximum (FWHM) bandwidth, 1\% response points, and the Integrated $\mathrm{OOB}$ (IOOB). J1 spectral characterization was completed successfully, and analysis completed by the government team based on the SNR and visual inspection led to the release of high quality J1 VIIRS RSR data set (Version 1, stitched in-band and OOB) in June 2015 [18]. Figure 7 shows the in-band RSR for VisNIR and DNB (top), SMWIR (middle) and LWIR (bottom). As noted previously, the redesign of the VisNIR filters led to major enhancements in terms of IOOB reduction as shown in Figure 8 where we compare the full M1 RSR (in-band and OOB) between J1 and SNPP sensors. Compliance with the band center, bandwidth, extended band-pass, and IOOB requirements were calculated for all bands on a per detector basis. Table 7 provides a band average summary of J1 spectral performance for all bands, showing non-compliances in shaded cells. As a comparison to SNPP VIIRS, performances that were not compliant for SNPP were underlined. We can easily observe the enhancements of J1 IOOB for the VisNIR bands compared to SNPP. Most other non-compliances are either similar to SNPP or not considered a risk to the SDR or EDR performance. Additional RSR testing was performed for VisNIR bands in the post-TV phase using the NIST laser source. Further enhancements to the VisNIR RSRs are expected before J1 instrument launch, which will be based on the combination of best quality measurements from SpMA and laser sources, and this new version is expected to be released in early 2016. 
Table 7. J1 Spectral performance (measurement $v$ s. specification) summary based on RSR Version 1 data set. Shaded cells represent J1 non-compliances. Underlined cells represent SNPP non-compliances.

\begin{tabular}{|c|c|c|c|c|c|c|c|c|c|c|}
\hline \multirow{2}{*}{ Band } & \multicolumn{2}{|c|}{ Center Wavelength $(\mathrm{nm})$} & \multicolumn{2}{|c|}{ Bandwidth (nm) } & \multicolumn{4}{|c|}{$1 \%$ Limits $(\mathrm{nm})$} & \multicolumn{2}{|c|}{ IOOB (\%) } \\
\hline & Meas. & $\begin{array}{c}\text { Spec. } \\
\text { Center/ } \\
\text { Tolerance }\end{array}$ & Meas. & \begin{tabular}{l}
\multicolumn{1}{c}{ Spec. } \\
Bandwidth/ \\
Tolerance
\end{tabular} & $\begin{array}{l}\text { Lower } \\
\text { Limit }\end{array}$ & $\begin{array}{l}\text { Upper } \\
\text { Limit }\end{array}$ & $\begin{array}{l}\text { Lower } \\
\text { Limit }\end{array}$ & $\begin{array}{l}\text { Upper } \\
\text { Limit }\end{array}$ & Meas. & Spec. \\
\hline I1 & 643.0 & $640 / 6$ & 78.6 & $80 / 6$ & 594.0 & 693.5 & 565 & 715 & 0.11 & 0.5 \\
\hline $\mathrm{I} 2$ & 867.3 & $865 / 8$ & 36.4 & $39 / 5$ & 841.6 & 893.5 & 802 & 928 & $\underline{0.12}$ & 0.7 \\
\hline I3 & 1603.2 & $1610 / 8$ & 60.4 & $60 / 5$ & 1544.5 & 1667.4 & 1509 & 1709 & $\overline{0.44}$ & 0.7 \\
\hline M1 & 411.2 & $412 / 2$ & 17.5 & $20 / 2$ & 395.3 & 425.4 & 376 & 444 & $\overline{0.24}$ & 1.0 \\
\hline M2 & 444.8 & $445 / 3$ & $\underline{17.0}$ & $18 / 2$ & 429.3 & 457.9 & 417 & 473 & $\overline{0.34}$ & 1.0 \\
\hline M3 & 488.6 & $488 / 4$ & $\overline{19.0}$ & $20 / 3$ & 473.0 & 504.4 & 455 & 521 & $\underline{0.30}$ & 0.7 \\
\hline M4 & $\underline{556.3}$ & $555 / 4$ & 18.5 & $20 / 3$ & 540.0 & 573.6 & 523 & 589 & $\underline{0.25}$ & 0.7 \\
\hline M5 & $\overline{667.1}$ & $672 / 5$ & 19.4 & $20 / 3$ & 649.6 & 684.9 & 638 & 706 & $\overline{0.27}$ & 0.7 \\
\hline M6 & 746.0 & $746 / 2$ & 13.5 & $15 / 2$ & 733.9 & 758.0 & 721 & 771 & $\overline{0.26}$ & 0.8 \\
\hline M12 & 3697.9 & $3700 / 32$ & 194.7 & $180 / 20$ & 3519.1 & 3893.7 & 3410 & 3990 & 0.36 & 1.1 \\
\hline M13 & 4073.9 & $4050 / 34$ & 154.9 & $155 / 20$ & 3911.7 & 4214.0 & 3790 & 4310 & 0.43 & 1.3 \\
\hline M14 & 8580.3 & $8550 / 70$ & $\underline{339.7}$ & $300 / 40$ & 8336.4 & 8879.1 & 8050 & 9050 & 0.23 & 0.9 \\
\hline M15 & 10730.8 & $10763 / 113$ & 1000.7 & $1000 / 100$ & 9915.8 & 11638.0 & 9700 & 11740 & $\underline{0.41}$ & 0.4 \\
\hline M16A & $\underline{11882.6}$ & $12013 / 88$ & 914.2 & $950 / 50$ & 11104.5 & 12692.3 & 11060 & 13050 & $\overline{0.46}$ & 0.4 \\
\hline M16B & $\overline{11883.0}$ & $12013 / 88$ & 933.8 & $950 / 50$ & 11101.8 & 12698.1 & 11060 & 13050 & $\overline{0.47}$ & 0.4 \\
\hline DNBMGS & $\overline{693.1}$ & $700 / 14$ & 380.5 & $400 / 20$ & 488.2 & 906.6 & 470 & 960 & $\overline{0.03}$ & 0.1 \\
\hline DNBLGS & 695.6 & $700 / 14$ & 380.3 & $400 / 20$ & 480.8 & 904.5 & 470 & 960 & 0.02 & 0.1 \\
\hline
\end{tabular}
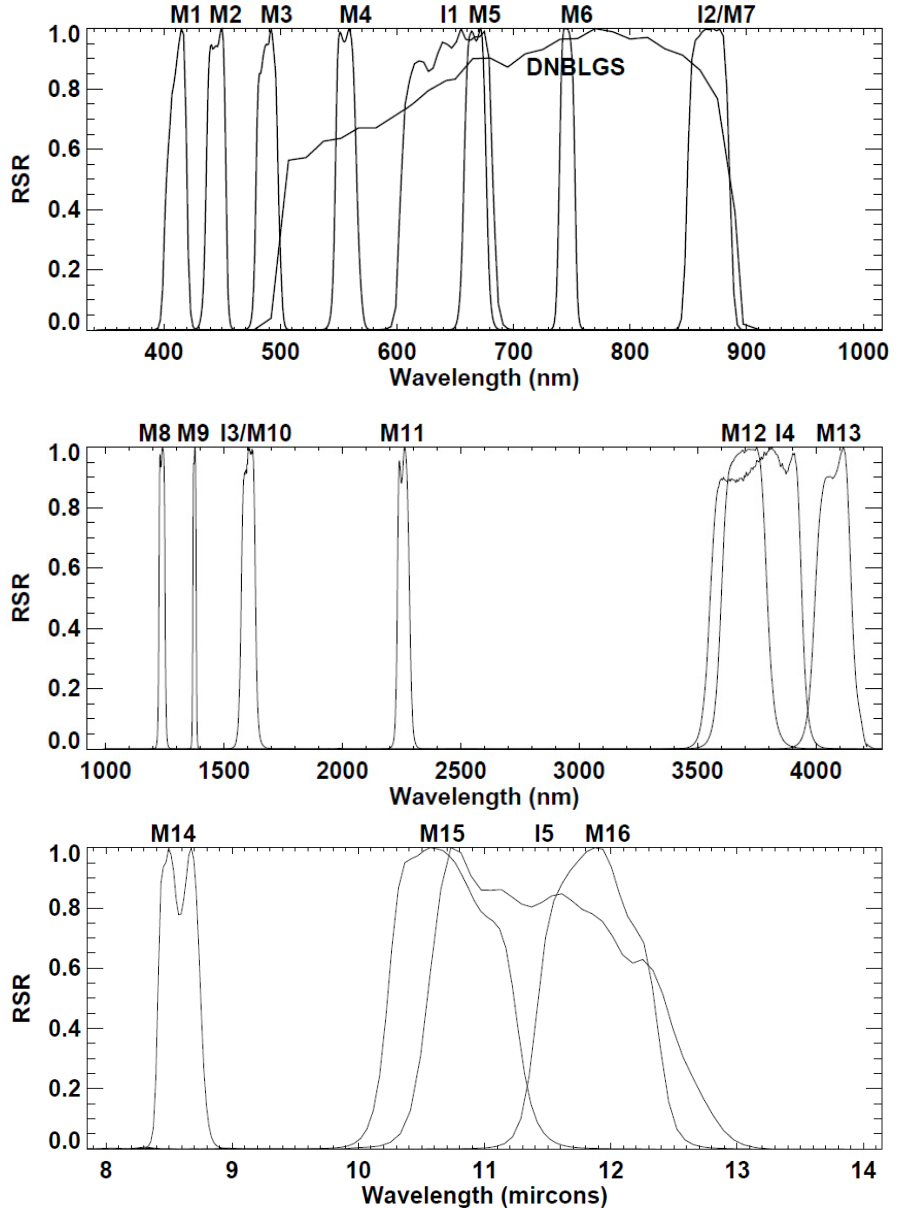

Figure 7. J1 VIIRS in-band RSRs, for VisNIR and DNB (top); SMWIR (middle); and LWIR (bottom). 


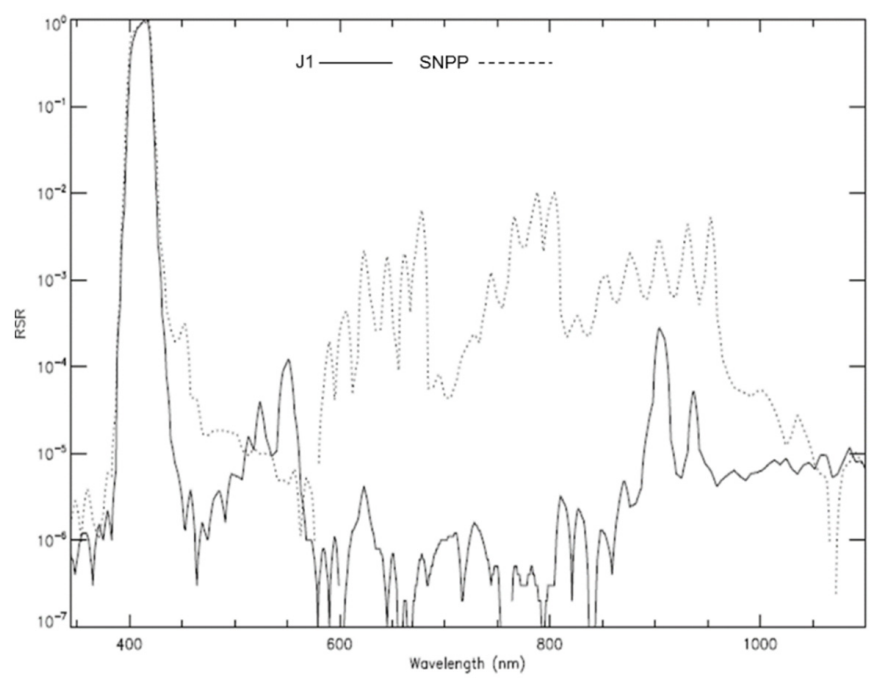

Figure 8. Full M1 RSR comparison between J1 (line) and SNPP (dashes).

\subsection{Response Versus Scan-Angle (RVS)}

The RVS testing was performed during ambient phase for the RSB using the SIS100 and for the TEB using the laboratory ambient blackbody (LABB) source and the on-board BB. Data taken at eleven angles-of-incidence (AOI) for the RSB and twelve for the TEB were used to fit the RVS function, which is a quadratic polynomial in AOI, after correcting for source drift and background radiances [19].

Figure 9 shows $\mathrm{J} 1$ band-average RVS functions for HAM side 0. For all bands, the RVS differences between HAM sides are small with the exception of bands M1, M2, M7 and I2. The variation in the RSB RVS for M1-M11 and I1-I3 is generally small (top and middle plots) and similar to SNPP, varying by less than $1.5 \%$ (M1 has largest variation) over the full operational AOI range of $28.6^{\circ}$ to $60.2^{\circ}$. The RSB RVS uncertainty target is $0.3 \%$ which was determined here as the average fitting residuals. All RSB bands had uncertainties lower than $0.06 \%$ with the highest uncertainty observed for DNB, and M9 due to error residuals from water vapor correction [20]. The only exception is I3 detector 4 (very noisy detector) which has shown an uncertainty of about $0.4 \%$.

The J1 band averaged RVS for the MWIR bands, M12-M13 and I4, and the LWIR bands, M14-M16 and I5, are plotted in the middle and bottom plots respectively of Figure 8. The MWIR RVS is generally small varying by less than $1 \%$ over the full AOI range. In contrast, the LWIR RVS changes by up to $10 \%$ for M14 over the range of AOI. The TEB RVS uncertainty target is $0.2 \%$, which was determined here as the average fitting residuals; all TEB RVS uncertainties are lower than $0.1 \%$, meeting the RVS characterization uncertainty target with good margin. Overall, J1 RVS performance is comparable to SNPP performance.

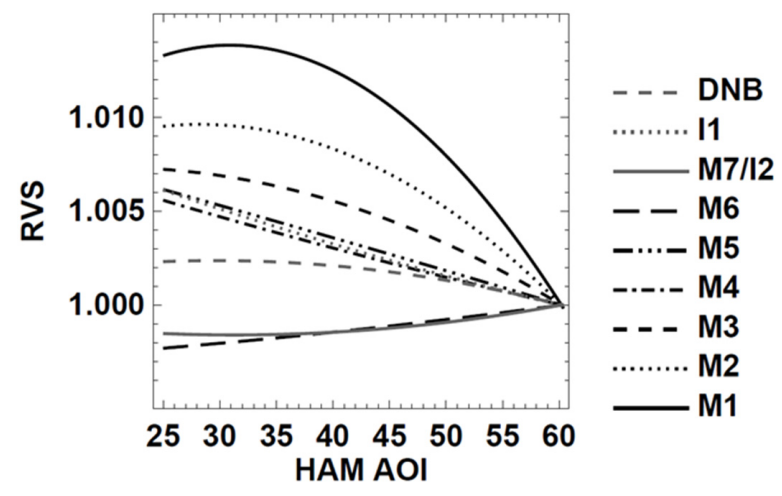

Figure 9. Cont. 

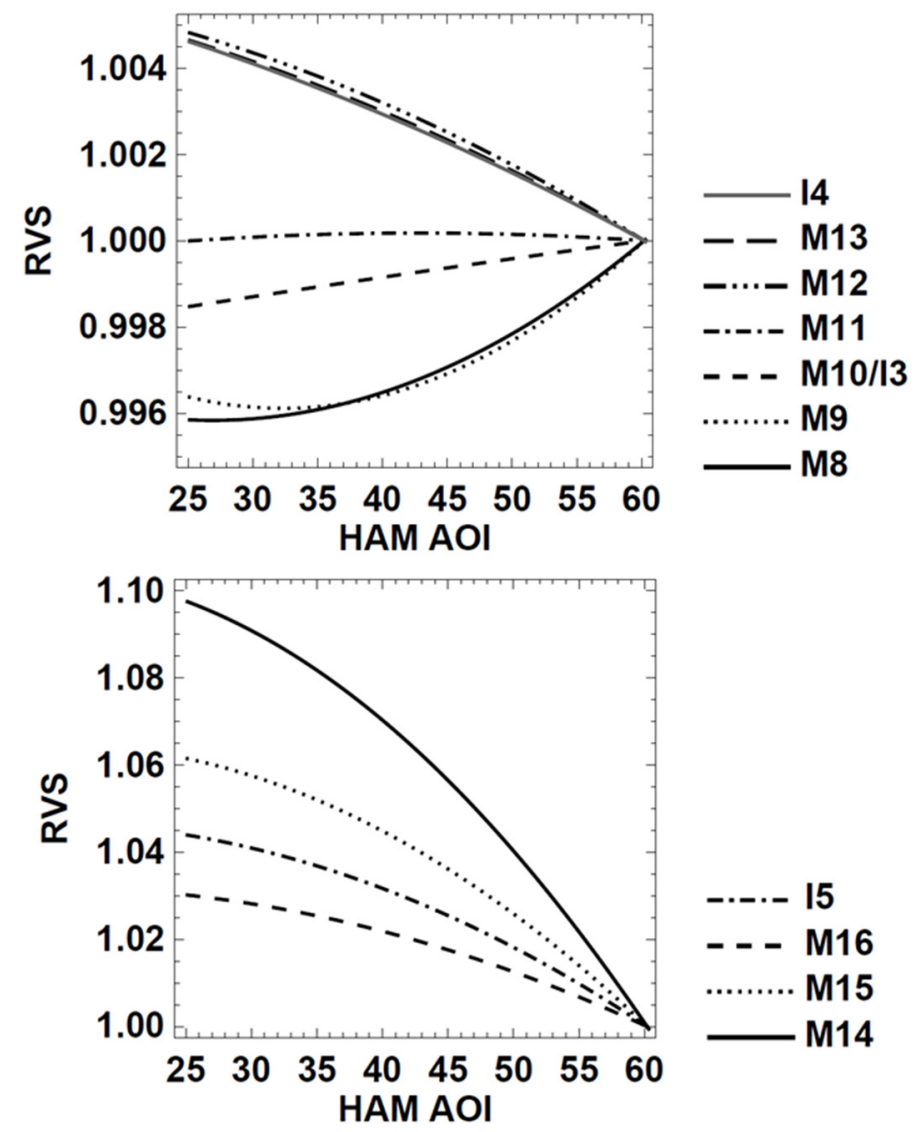

Figure 9. J1 VIIRS band averaged RVS as a function of HAM AOI for HAM 0.

\subsection{Near-Field Response (NFR)}

NFR is defined as scattered light originating from within 4 degrees of the RTA line of sight. The Scatter Measurement Assembly (ScMA) light source was used with a slit and a band-pass filter to measure the sensor NFR. The VIIRS NFR is limited by the absolute radiometric calibration uncertainty requirement for structured scenes which sets the maximum allowable response at a specified angle limit coming off a 12 by 12 milliradian bright target for each band. To estimate the structured scene response, a Harvey-Shack BRDF scattering model [21,22] was used to fit the measured NFR profile and remove test artifacts and noisy samples.

Figure 10 compares the normalized response for band M5 detector 8 between J1 and SNPP, which represents a typical profile observed in the VIIRS NFR measurements when the sensor is scanning the source through a vertical slit reticle. For both SNPP and J1, the figure shows the NFR falling off rapidly from the peak with additional sharp drops seen around the field baffle locations. In this example, the M5 NFR drops to about $0.1 \%$ within 5 samples of the peak (i.e., corresponding to approximately $1.25 \mathrm{~km}$ at the Earth surface), and the field baffle reduces the NFR by about one order of magnitude. The NFR is estimated for each detector, and the results show good detector uniformity within the same band. The NFR requirement states that the maximum allowable scattered radiance, as a fraction of typical scene radiance, at the specified angular distance from a bright target shall be less than the specified value. The band averaged VIIRS NFR performance is summarized in Table 8 , including the bright target radiance $\left(\mathrm{L}_{\text {bright }}\right)$, the specification $\left(\mathrm{L}_{\mathrm{spec}}\right)$, and the ratio $\mathrm{L}_{\text {scat }} / / \mathrm{L}_{\mathrm{spec}}$ for J1 and SNPP VIIRS. The results show all J1 VIIRS bands meet the specification $\left(\mathrm{L}_{\mathrm{scat}} / \mathrm{L}_{\mathrm{spec}}<1\right)$ with margin at the beginning of life, and multiple bands are showing performance enhancements when compared to SNPP, especially M15 and M16. 


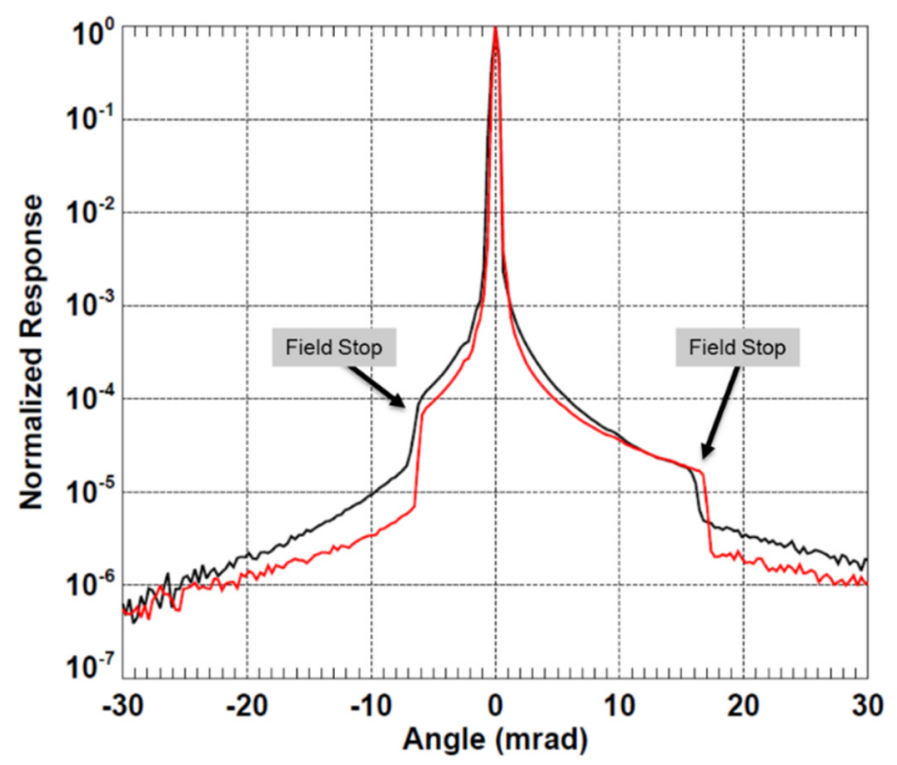

Figure 10. Measured NFR for band M5 $(672 \mathrm{~nm})$ detector 8 as a function of scan angle, using J1 data (red line) and SNPP data (black line).

Table 8. The summary of J1 band average NFR performance and comparison to SNPP. The ratio $\mathrm{L}_{\text {scat }} / \mathrm{L}_{\text {spec }}$ less than unity means compliance with scatter requirements.

\begin{tabular}{|c|c|c|c|c|c|c|c|}
\hline \multirow{2}{*}{ Band } & \multirow{2}{*}{$\begin{array}{c}\text { Center } \\
\text { Wavelength }(\mathrm{nm})\end{array}$} & \multirow{2}{*}{$\begin{array}{c}\text { Angular } \\
\text { Separation (mrad) }\end{array}$} & \multirow{2}{*}{$\mathbf{L}_{\text {bright }}$} & \multicolumn{2}{|c|}{ SNPP } & \multicolumn{2}{|c|}{$\mathrm{J1}$} \\
\hline & & & & $\mathbf{L}_{\text {spec }}$ & $\mathrm{L}_{\text {scat }} / \mathrm{L}_{\text {spec }}$ & $\mathbf{L}_{\text {spec }}$ & $\mathrm{L}_{\text {scat }} / \mathrm{L}_{\text {spec }}$ \\
\hline M1 & 412 & 6 & 162 & $2.77 \times 10^{-3}$ & 0.39 & $4.68 \times 10^{-3}$ & 0.37 \\
\hline M3 & 488 & 6 & 160 & $2.00 \times 10^{-3}$ & 0.5 & $3.33 \times 10^{-3}$ & 0.36 \\
\hline M4 & 555 & 6 & 160 & $1.31 \times 10^{-3}$ & 0.47 & $2.25 \times 10^{-3}$ & 0.48 \\
\hline M5 & 672 & 6 & 115 & $8.70 \times 10^{-4}$ & 0.63 & $9.55 \times 10^{-4}$ & 0.67 \\
\hline M6 & 746 & 12 & 147 & $1.31 \times 10^{-3}$ & 0.12 & $1.08 \times 10^{-3}$ & 0.13 \\
\hline M8 & 1240 & 6 & 57 & $9.47 \times 10^{-4}$ & 0.62 & $4.92 \times 10^{-4}$ & 0.65 \\
\hline M9 & 1378 & NA & NA & NA & NA & NA & NA \\
\hline M10 & 1610 & 6 & 86.1 & $8.48 \times 10^{-4}$ & 0.76 & $1.03 \times 10^{-3}$ & 0.38 \\
\hline M11 & 2250 & 6 & 1.2 & $1.00 \times 10^{-3}$ & 0.42 & $6.03 \times 10^{-6}$ & 0.63 \\
\hline M12 & 3700 & 3 & 0.3 & $1.67 \times 10^{-3}$ & 0.64 & $4.78 \times 10^{-4}$ & 0.87 \\
\hline M13 & 4050 & 3 & 1.7 & $1.86 \times 10^{-3}$ & 0.63 & $3.15 \times 10^{-3}$ & 0.47 \\
\hline M14 & 8550 & NA & NA & NA & NA & NA & NA \\
\hline
\end{tabular}

\subsection{Stray Light Response (SLR)}

The far field stray light is defined as the light originating from the region between 4 and 62.5 degrees from the sensor boresight. The far field stray light testing was performed using a radiometrically calibrated $1000 \mathrm{~W}$ studio lamp. The VIIRS telescope was staring at a cavity type blackbody while the lamp was moved through 33 equally distanced hemispherical positions to evaluate the angular stray light distribution. The lamp positions covered roughly equally divided annulus out to 62.5 degrees off nadir to simulate the Earth as subtended by VIIRS at the J1 on-orbit operating altitude. The measured instrument response was scaled by the ratio of the studio lamp irradiance and a model of bright cloud irradiance. The VIIRS far-field stray light testing represent a worse case, hole-in-the-cloud scenario. The measured instrument response at each position was weighted by the corresponding annulus, then aggregated to estimate the total stray light contribution from the specified worse-case scenario. The test was conducted with the RTA locked at SV, nadir, and end-of-scan to obtain stray light estimates at different scan angles. 
Table 9 shows the estimated J1 VIIRS far field stray light compliance. The sensor test data analysis showed all RSB meet the stray light requirement with large margins at the beginning of life. Margins are between $45 \%$ (M5) and $94 \%$ (M10, I3). Furthermore, test results indicate that the stray light rejection is comparable between J1 and SNPP. A noticeable difference is that the stray light in SNPP is much stronger along track than along scan direction; whereas in J1, the stray light is more evenly distributed over all angles. Another difference is that band M11 is now compliant for J1 because of the $\mathrm{L}_{\text {typ }}$ requirement change (from 0.1 to $1.0 \mathrm{Wm}^{-2} \cdot \mathrm{sr}^{-1} \cdot \mu \mathrm{m}^{-1}$ ).

Table 9. Summary of J1 VIIRS stray light performance, and the ratio of measured stray light to requirement $\left(1 \% \mathrm{~L}_{\text {typ }}\right)$. The comparison to SNPP stray light performance is also shown.

\begin{tabular}{|c|c|c|c|c|c|c|c|c|}
\hline Band & $\mathrm{E}_{\text {earth }}$ & $\mathrm{L}_{\text {typ }}$ & \multicolumn{3}{|c|}{ SNPP } & \multicolumn{3}{|c|}{ J1 } \\
\hline M1 & 1444.1 & 44.9 & 17.02 & 2.58 & 0.15 & 7.84 & 2.42 & 0.31 \\
\hline M3 & 1563.5 & 32 & 8.81 & 2.56 & 0.29 & 8.12 & 2.89 & 0.36 \\
\hline M4 & 1510.7 & 21 & 7.53 & 2.39 & 0.32 & 7.50 & 2.96 & 0.39 \\
\hline M5 & 1265.7 & 10 & 5.06 & 2.38 & 0.47 & 4.69 & 2.57 & 0.55 \\
\hline M7 & 833.2 & 6.4 & 6.87 & 4.06 & 0.59 & 6.27 & 3.29 & 0.52 \\
\hline M8 & 353 & 5.4 & 1.45 & 0.77 & 0.53 & 1.82 & 0.44 & 0.24 \\
\hline M9 & 262.9 & 6 & 2.44 & 0.92 & 0.38 & 2.41 & 0.36 & 0.15 \\
\hline M10 & 165.7 & 7.3 & 3.55 & 1.30 & 0.36 & 3.44 & 0.22 & 0.06 \\
\hline M11 & 56.4 & 0.12 & 0.11 & 0.42 & 3.77 & 1.15 & 0.09 & 0.08 \\
\hline I1 & 1341.3 & 22 & 1.02 & 0.26 & 0.26 & 0.94 & 0.31 & 0.33 \\
\hline
\end{tabular}

\section{Conclusions}

This paper provides an early assessment of the J1 VIIRS pre-launch radiometric performance, based on an intensive and comprehensive testing program that was designed by the sensor contractor with support from the government calibration and science teams. The instrument testing program was performed to characterize the radiometric, spectral and spatial performance for all 22 spectral bands in various configurations and environments, and simulating the range of on-orbit conditions (ambient and thermal vacuum). The key radiometric and spectral performances were discussed in this paper. The VIIRS spectral bands calibration and characterization have shown very good performance, providing good quality calibration data to populate the SDR LUTs. All performance non-compliances and features were investigated, understood, and characterized using pre-launch test data and/or sensor model simulations. The RSB SNR was compliant for all bands with minimum margin of $51 \%$ (M2) and maximum margin of $3172 \%$ (I3), while the dynamic range was compliant for all RSB except bands M8 (72\%) and I3 (91\%). As expected, and similar to SNPP, the TEB have shown very good performance in term of NEdT and dynamic range (full compliance). The spectral testing also provided good quality data to generate the sensor RSRs, and the minor spectral non-compliances are band center for M16, bandpass for M1 and M8, 1\% upper limit for I5, and IOOB for M15 and M16. All of these spectral non-compliances are expected to have negligible impact on the data products. The RVS derived for J1 was as expected, very similar to SNPP, and meeting the uncertainty target of $0.3 \%$ for RSB and $0.2 \%$ for TEB. The scattered light performance represented by the NFR and SLR are meeting the sensor requirements for all bands, and are expected to lead to better or similar performance as SNPP. Overall, J1 performance was comparable to SNPP VIIRS, and it is expected to have high quality J1 data product post-launch. Two minor issues related to high polarization sensitivity (M1-M4) and high non-linearity at low radiance for the DNB and SWIR bands were well characterized, and necessary 
mitigation plans are being prepared within the ground processing system to generate high quality products on-orbit.

Acknowledgments: The authors of this paper would like to thank the VIIRS team members from Raytheon, NOAA, Aerospace Corporation, NASA Science Team, and University of Wisconsin team for their valuable contributions to VIIRS testing and performance verifications. We also want to thank James McCarthy (Stellar Solutions), David Moyer (Aerospace Corporation), Chris Moeller (University of Wisconsin), and previous VCST members for their valuable support to the VIIRS program and to the pre-launch calibration and characterization effort.

Author Contributions: Hassan Oudrari was the lead author who provided the outline of the paper, contributed to the introduction, the sensor design and the sensor testing, and the compilation of the final paper. Jeff McIntire provided the thermal emissive bands performance assessment, the response versus scan-angle performance, and the polarization sensitivity assessment. Xiaoxiong Xiong provided contribution to the sensor design and testing description, and the review of the paper at multiple phases to enhance quality and consistency. James Butler provided the contribution to the sensor design and testing description, and the review of the paper at multiple phases to enhance quality and consistency. Qiang Ji provided the reflective solar bands performance, the stray light performance, and near field performance assessments. Thomas Schwarting provided the relative spectral response assessments, and contributions to the response versus scan-angle. Shihyan Lee provided contributions to the DNB performance, the reflective solar bands performance, response versu scan-angle performance assessments. Boryana Efremova provided assessments to the thermal emissive bands performance assessments.

Conflicts of Interest: The authors declare no conflict of interest.

\section{References}

1. Cao, C.; de Luccia, F.J.; Xiong, X.; Wolfe, R.; Weng, F. Early On-Orbit Performance of the Visible Infrared Imaging Radiometer Suite Onboard the Suomi National Polar-Orbiting Partnership(S-NPP) Satellite. IEEE Trans. Geosci. Remote Sens. 2013, 52, 1142-1156. [CrossRef]

2. Xiong, X.; Butler, J.; Chiang, K.; Efremova, B.; Fulbright, J.; Lei, N.; McIntire, J.; Oudrari, H.; Sun, J.; Wang, Z.; et al. VIIRS on-orbit calibration methodology and performance. J. Geophys. Res. Atmos. 2014, 119, 5065-5078. [CrossRef]

3. Jackson, J.; Liu, H.; Laszlo, I.; Kondragunta, S.; Remer, L.A.; Huang, J.; Huang, H.-C. Suomi-NPP VIIRS Aerosol Algorithms and Data Products. J. Geophys. Res. Atmos. 2013, 118, 12,673-12,689. [CrossRef]

4. Justice, C.O.; Román, M.; Csiszar, I.; Vermote, E.F.; Wolfe, R.E.; Hook, S.J.; Friedl, M.; Wang, Z.; Schaaf, C.B.; Miura, T.; et al. Land and cryosphere products from Suomi NPP VIIRS: Overview and status. J. Geophys. Res. Atmos. 2013, 118, 9753-9765. [CrossRef] [PubMed]

5. Wang, M.; Liu, X.; Tan, L.; Jiang, L.; Son, S.; Shi, W.; Rausch, K.; Voss, K. Impacts of VIIRS SDR performance on ocean color products. J. Geophys. Res. Atmos. 2013, 118, 10347-10360. [CrossRef]

6. Barnes, W.; Salomonson, V. MODIS: A global image spectroradiometer for the earth observing system. Crit. Rev. Opt. Sci. Technol. 1993, 47, 285-307.

7. McClain, C.; Hooker, S.; Feldman, G.; Bontempi, P. Satellite data for ocean biology, biogeochemistry, and climate research. Eos Trans. AGU 2006, 87, 337-339. [CrossRef]

8. King, M.D.; Menzel, W.P.; Kaufman, Y.J.; Tanre, D.; Gao, B.-C.; Platnick, S.; Ackerman, S.A.; Remer, L.A.; Pincus, R.; Hubanks, P.A. Cloud and aerosol and water vapor properties, precipitable water, and profiles of temperature and humidity from MODIS. IEEE Trans. Geosci. Remote Sen. 2003, 41, 442-458. [CrossRef]

9. Justice, C.O.; Vermote, E.; Townshend, J.R.G.; Defries, R.; Roy, D.P.; Hall, D.K.; Salomonson, V.V.; Privette, J.L.; Riggs, G.; Strahler, A.; et al. The moderate resolution imaging spectroradiometer (MODIS): Land remote sensing for global change research. IEEE Trans. Geosci. Remote Sen. 1998, 36, 1228-1249. [CrossRef]

10. Cao, C.; Xiong, X.; de Luccia, F.; Liu, Q.; Blonski, S.; Pogorzala, D.; Oudrari, H. VIIRS SDR User's Guide; Tech. Rep. NESDIS 142; U.S. Dept. Commerce, NOAA: Silver Spring, MD, USA, Febuary 2013.

11. Oudrari, H.; McIntire, J.; Xiong, X.; Butler, J.; Lee, S.; Lei, N.; Schwarting, T.; Sun, J. Prelaunch Radiometric Characterization and Calibration of the S-NPP VIIRS Sensor. IEEE Trans. Geosci. Remote Sen. 2015, 53, 2195-2210. [CrossRef]

12. Joint Polar Satellite System (JPSS). VIIRS General Test Plan (GTP); Goddard Space Flight Center: Greenbelt, MD, USA, 2011.

13. Joint Polar Satellite System (JPSS). VIIRS SDR Algorithm Theoretical Basis Document (ATBD); Goddard Space Flight Center: Greenbelt, MD, USA, 2011. 
14. Joint Polar Satellite System (JPSS). VIIRS Reflective Solar Bands-Performance Verification Report (PVR); Goddard Space Flight Center: Greenbelt, MD, USA, 2011.

15. McIntire, J.; Moyer, D.; Oudrari, H.; Xiaoxiong, X. Pre-launch radiometric characterization of JPSS-1 VIIRS thermal emissive bands. Remote Sens. 2015. in press.

16. McIntire, J.; Young, J.B.; Moyer, D.; Waluschka, E.; Oudrari, H.; Xiong, X. Analysis of JPSS J1 VIIRS Polarization Sensitivity Using the NIST T-SIRCUS. Proc. SPIE 2015, 9607, 960713.

17. Waluschka, E.; McCorkel, J.; McIntire, J.; Moyer, D.; McAndrew, B.; Brown, S.W.; Lykke, K.; Young, J.B.; Fest, E.; Butler, J.; et al. J1 VIIRS polarization narrative: testing and performance. Proc. SPIE 2015, 9607, 960712.

18. Moeller, C.; Schwarting, T.; McIntire, J.; Moyer, D. JPSS-1 VIIRS pre-launch spectral characterization and performance. Proc. SPIE 2015, 9607, 960711.

19. Moyer, D.; McIntire, J.; Oudrari, H.; McCarthy, J.; Xiaosiong, X.; de Luccia, F. JPSS-1 VIIRS pre-launch response versus scan-angle testing and performance. Remote Sens. 2015, in press.

20. Lee, S.; Cao, C. JPSS-1 VIIRS Prelaunch RSB/DNB RVS characterization and water vapor correction. Proc. SPIE 2015, 9607, 96071R.

21. Joint Polar Satellite System (JPSS). VIIRS Product Requirement Document (PRD); Goddard Space Flight Center: Greenbelt, MD, USA, 2011.

22. Joint Polar Satellite System (JPSS). J1 VIIRS Near Field Response (NFR); Performance Verification Report (PVR); Goddard Space Flight Center: Greenbelt, MD, USA, 2011.

(C) 2016 by the authors; licensee MDPI, Basel, Switzerland. This article is an open access article distributed under the terms and conditions of the Creative Commons by Attribution (CC-BY) license (http:/ / creativecommons.org/licenses/by/4.0/). 